\title{
Derived deformations of schemes
}

\author{
Jonathan PAul PRidham
}

We introduce a new approach to constructing derived deformation groupoids, by considering them as parameter spaces for strong homotopy bialgebras. This allows them to be constructed for all classical deformation problems, such as deformations of an arbitrary scheme, in any characteristic. We also give a general approach for studying deformations of diagrams.

\section{Introduction}

1 Derived deformation functors

1.1 Simplicial Artinian rings

1.2 Properties of morphisms

1.3 Derived deformation functors

1.4 Quotient spaces

1.5 Cohomology and obstructions

1.6 Model structures

1.6.1 Homotopy representability

2 Monads and comonads

2.2 Bialgebras 
3.2 SDCs from diagrams

3.3 Constrained deformations

4 Extended deformation functors from SDCs

4.1 Deformations of morphisms

4.1.1 Deforming identity morphisms

5 Comparison with [11]

5.1 DGLAs

556

5.2 Cosimplicial groups

5.3 The final comparison

559

5.4 An application

561

Acknowledgments

562

References

562

\section{Introduction}

In [11], the theory of simplicial deformation complexes (SDCs) was expounded as a means of governing deformation problems, giving an alternative to the theory of differential graded Lie algebras (DGLAs). The main advantages of SDCs over DGLAs are that they can be constructed canonically (and thus for a wider range of problems), and are valid in all characteristics.

In [9], Manetti showed that given a DGLA, or even an SHLA, governing a deformation problem, it is possible to define an extended deformation functor. The approach in this paper can almost be regarded as opposite to this - we try, for any deformation problem, to define an extended deformation functor with a geometric interpretation, meaning that the functor still parametrizes geometric objects. We then see how this functor can be recovered from the SDC governing the problem.

Since almost all examples of SDCs come from monadic and comonadic adjunctions, in Section 4 we look at how to extend deformation groupoids 
in these scenarios. For a monad $T$, the solution is to look at the strong homotopy $T$-algebras, as defined by Lada in [1]. The idea is that the monadic axioms are only satisfied up to homotopy, with the homotopies satisfying further conditions up to homotopy, and so on. This approach allows us to define a quasi-smooth extended deformation functor associated to any SDC, with the same cohomology.

Using the constructions of Sections 3.2 and 3.3, we describe extended deformations of morphisms and diagrams (giving new results even for the problems in [11]). This defines cohomology of a morphism in any such category, giving a variant of Van Osdol's bicohomology [17]. One consequence is that the space describing extended deformations of the identity morphism on an object $D$ is just the loop space of the space of extended deformations of $D$.

The structure of the paper is as follows. Sections 1 and 2 are introductory, summarizing results from [14] and properties of monads and comonads, respectively. Section 3 reprises material from [11] on SDCs, and includes new results constructing SDCs associated to diagrams in Sections 3.2 and 3.3. The key motivating examples of deformations of a scheme are described in Examples 3.1 and 3.4.

Section 4 then gives the construction of the derived deformation functor (Definition 4.2), together with a simplified description of derived deformations of a morphism (Proposition 4.6), and the characterization of derived deformations of an identity morphism as a loop space (Proposition 4.7).

In [11], it was shown that SDCs are equivalent to $\mathbb{N}_{0}$-graded DGLAs in characteristic 0 , in such a way that the associated deformation groupoids are equivalent. In Section 5, we show that the associated extended deformation functors are also equivalent. Corollary 5.4 then shows how our functor of derived deformations of a smooth scheme coincides with existing constructions.

\section{Derived deformation functors}

With the exception of Section 1.4, the definitions and results in this section can all be found in [14]. Fix a complete local Noetherian ring $\Lambda$, with maximal ideal $\mu$ and residue field $k$.

\subsection{Simplicial Artinian rings}

Definition 1.1. Let $\mathcal{C}_{\Lambda}$ denote the category of local Artinian $\Lambda$-algebras with residue field $k$. We define $s \mathcal{C}_{\Lambda}$ to be the category of Artinian simplicial local $\Lambda$-algebras, with residue field $k$. 
Definition 1.2. Given a simplicial complex $V_{\bullet}$, recall that the normalized chain complex $N^{s}(V) \bullet$ is given by $N^{s}(V)_{n}:=\bigcap_{i>0} \operatorname{ker}\left(\partial_{i}: V_{n} \rightarrow\right.$ $\left.V_{n-1}\right)$, with differential $\partial_{0}$. The simplicial Dold-Kan correspondence says that $N^{s}$ gives an equivalence of categories between simplicial complexes and non-negatively graded chain complexes in any abelian category. Where no ambiguity results, we will denote $N^{s}$ by $N$.

Lemma 1.1. A simplicial complex $A_{\bullet}$ of local $\Lambda$-algebras with residue field $k$ and maximal ideal $\mathfrak{m}(A)$ • is Artinian if and only if:

(1) the normalization $N(\cot A)$ of the cotangent space $\cot A:=\mathfrak{m}(A) /$ $\left(\mathfrak{m}(A)^{2}+\mu \mathfrak{m}(A)\right)$ is finite dimensional (i.e., concentrated in finitely many degrees, and finite dimensional in each degree).

(2) For some $n>0, \mathfrak{m}(A)^{n}=0$.

Proof. See [14, Lemma 1.16]

As in [4], we say that a functor is left exact if it preserves all finite limits. This is equivalent to saying that it preserves final objects and fibre products.

Definition 1.3. Define Sp to be the category of left-exact functors from $\mathcal{C}_{\Lambda}$ to Set. Define $c \mathrm{Sp}$ to be the category of left-exact functors from $s \mathcal{C}_{\Lambda}$ to Set.

Definition 1.4. Given a functor $F: \mathcal{C}_{\Lambda} \rightarrow$ Set, we write $F: s \mathcal{C}_{\Lambda} \rightarrow$ Set to mean $A \mapsto F\left(A_{0}\right)$ (corresponding to the inclusion $\mathrm{Sp} \hookrightarrow c \mathrm{Sp}$ ).

\subsection{Properties of morphisms}

Definition 1.5. As in [8], we say that a functor $F: \mathcal{C}_{\Lambda} \rightarrow$ Set is smooth if for all surjections $A \rightarrow B$ in $\mathcal{C}_{\Lambda}$, the map $F(A) \rightarrow F(B)$ is surjective.

Definition 1.6. We say that a map $f: A \rightarrow B$ in $s \mathcal{C}_{\Lambda}$ is acyclic if $\pi_{i}(f)$ : $\pi_{i}(A) \rightarrow \pi_{i}(B)$ is an isomorphism of Artinian $\Lambda$-modules for all $i . f$ is said to be surjective if each $f_{n}: A_{n} \rightarrow B_{n}$ is surjective.

Note that for any simplicial abelian group $A$, the homotopy groups can be calculated by $\pi_{i} A \cong H_{i}(N A)$, the homology groups of the normalized chain complex. These in turn are isomorphic to the homology groups of the unnormalized chain complex associated to $A$. 
Definition 1.7. We define a small extension $e: I \rightarrow A \rightarrow B$ in $s \mathcal{C}_{\Lambda}$ to consist of a surjection $A \rightarrow B$ in $s \mathcal{C}_{\Lambda}$ with kernel $I$, such that $\mathfrak{m}(A) \cdot I=0$. Note that this implies that $I$ is a simplicial complex of $k$-vector spaces.

Lemma 1.2. Every surjection in $s \mathcal{C}_{\Lambda}$ can be factorized as a composition of small extensions. Every acyclic surjection in $s \mathcal{C}_{\Lambda}$ can be factorized as a composition of acyclic small extensions.

Proof. See [14, Lemma 1.23].

Definition 1.8. We say that a morphism $\alpha: F \rightarrow G$ in $c \mathrm{Sp}$ is smooth if for all small extensions $A \rightarrow B$ in $s \mathcal{C}_{\Lambda}$, the map $F(A) \rightarrow F(B) \times_{G(B)} G(A)$ is surjective.

Similarly, we call $\alpha$ quasi-smooth if for all acyclic small extensions $A \rightarrow$ $B$ in $s \mathcal{C}_{\Lambda}$, the map $F(A) \rightarrow F(B) \times_{G(B)} G(A)$ is surjective.

Lemma 1.3. A morphism $\alpha: F \rightarrow G$ in $\mathrm{Sp}$ is smooth if and only if the induced morphism between the objects $F, G \in c \mathrm{Sp}$ is quasi-smooth, if and only if it is smooth.

Proof. See [14, Lemma 1.31].

\subsection{Derived deformation functors}

Definition 1.9. Define the $s c \mathrm{Sp}$ to be the category of left-exact functors from $s \mathcal{C}_{\Lambda}$ to the category $\mathbb{S}$ of simplicial sets.

Definition 1.10. A morphism $\alpha: F \rightarrow G$ in $s c S p$ is said to be smooth if

(S1) for every acyclic surjection $A \rightarrow B$ in $s \mathcal{C}_{\Lambda}$, the map $F(A) \rightarrow F(B) \times_{G(B)}$ $G(A)$ is a trivial fibration in $\mathbb{S}$;

(S2) for every surjection $A \rightarrow B$ in $s \mathcal{C}_{\Lambda}$, the map $F(A) \rightarrow F(B) \times_{G(B)} G(A)$ is a surjective fibration in $\mathbb{S}$.

A morphism $\alpha: F \rightarrow G$ in $s c \mathrm{Sp}$ is said to be quasi-smooth if it satisfies (S1) and

(Q2) for every surjection $A \rightarrow B$ in $s \mathcal{C}_{\Lambda}$, the map $F(A) \rightarrow F(B) \times_{G(B)} G(A)$ is a fibration in $\mathbb{S}$. 
Definition 1.11. Given $A \in s \mathcal{C}_{\Lambda}$ and a finite simplicial set $K$, define $A^{K} \in$ $\mathcal{C}_{\Lambda}$ by

$$
\left(A^{K}\right)_{i}:=\operatorname{Hom}_{\mathbb{S}}\left(K \times \Delta^{i}, A\right) \times_{\operatorname{Hom}_{\text {Set }}\left(\pi_{0} K, k\right)} k .
$$

Definition 1.12. Given $F \in s c \operatorname{Sp}$, define $\underline{F}: s \mathcal{C}_{\Lambda} \rightarrow \mathbb{S}$ by

$$
\underline{F}(A)_{n}:=F_{n}\left(A^{\Delta^{n}}\right) \text {. }
$$

For $F \in c \mathrm{Sp}$, we may regard $F$ as an object of $s c \mathrm{Sp}$ (with the constant simplicial structure), and then define $\underline{F}$ as above.

Lemma 1.4. A map $\alpha: F \rightarrow G$ in $c \mathrm{Sp}$ is smooth (resp. quasi-smooth) if and only if the induced map of functors $\underline{\alpha}: \underline{F} \rightarrow \underline{G}$ is smooth (resp. quasismooth) in scSp.

Proof. See [14, Lemma 1.36].

The following lemma will provide many examples of functors which are quasi-smooth but not smooth.

Lemma 1.5. If $F \rightarrow G$ is a quasi-smooth map of functors $F, G: s \mathcal{C}_{\Lambda} \rightarrow \mathbb{S}$, and $K \rightarrow L$ is a cofibration in $\mathbb{S}$, then

$$
F^{L} \rightarrow F^{K} \times{ }_{G^{K}} G^{L}
$$

is quasi-smooth.

Proof. This is an immediate consequence of the fact that $\mathbb{S}$ is a simplicial model category, following from axiom SM7, as given in [3, Section II.3].

The following lemma is a consequence of standard properties of fibrations and trivial fibrations in $\mathbb{S}$.

Lemma 1.6. If $F \rightarrow G$ is a quasi-smooth map of functors $F, G: s \mathcal{C}_{\Lambda} \rightarrow \mathbb{S}$, and $H \rightarrow G$ is any map of functors, then $F \times_{G} H \rightarrow H$ is quasi-smooth.

Definition 1.13. A map $\alpha: F \rightarrow G$ of functors $F, G: \mathcal{C}_{\Lambda} \rightarrow \mathbb{S}$ is said to be smooth (resp. quasi-smooth, resp. trivially smooth) if for all surjections $A \rightarrow B$ in $\mathcal{C}_{\Lambda}$, the maps

$$
F(A) \rightarrow F(B) \times_{G(B)} G(A)
$$

are surjective fibrations (resp. fibrations, resp. trivial fibrations). 
Proposition 1.1. A map $\alpha: F \rightarrow G$ of left-exact functors $F, G: \mathcal{C}_{\Lambda} \rightarrow \mathbb{S}$ is smooth if and only if the maps $F_{n} \stackrel{\alpha_{n}}{\longrightarrow} G_{n}$ of functors $F_{n}, G_{n}: \mathcal{C}_{\Lambda} \rightarrow$ Set are all smooth.

Proof. See [14, Proposition 1.39].

Proposition 1.2. If a morphism $F \stackrel{\alpha}{\rightarrow} G$ of left-exact functors $F, G: s \mathcal{C}_{\Lambda} \rightarrow$ $\mathbb{S}$ is such that the maps

$$
\theta: F(A) \rightarrow F(B) \times_{G(B)} G(A)
$$

are surjective fibrations for all acyclic small extensions $A \rightarrow B$, then $\underline{\alpha}$ : $\underline{F} \rightarrow \underline{G}$ is quasi-smooth (resp. smooth) if and only if $\theta$ is a fibration (resp. surjective fibration) for all small extensions $A \rightarrow B$.

Proof. See [14, Proposition 1.63].

Definition 1.14. We will say that a morphism $\alpha: F \rightarrow G$ of quasi-smooth objects of $s c \mathrm{Sp}$ is a weak equivalence if, for all $A \in s \mathcal{C}_{\Lambda}$, the maps $\pi_{i} F(A) \rightarrow$ $\pi_{i} G(A)$ are isomorphisms for all $i$.

\subsection{Quotient spaces}

Definition 1.15. Given functors $X: s \mathcal{C}_{\Lambda} \rightarrow \mathbb{S}$ and $G: s \mathcal{C}_{\Lambda} \rightarrow s \mathrm{Gp}$, together with a right action of $G$ on $X$, define the quotient space by

$$
[X / G]_{n}=\left(X \times{ }^{G} W G\right)_{n}=X_{n} \times G_{n-1} \times G_{n-2} \times \cdots \times G_{0},
$$

with operations as standard for universal bundles (see [3, Chapter V]). Explicitly

$$
\begin{aligned}
& \partial_{i}\left(x, g_{n-1}, g_{n-2}, \ldots, g_{0}\right) \\
& \quad= \begin{cases}\left(\partial_{0} x * g_{n-1}, g_{n-2}, \ldots, g_{0}\right), & i=0 \\
\left(\partial_{i} x, \partial_{i-1} g_{n-1}, \ldots,\left(\partial_{0} g_{n-i}\right) g_{n-i-1}, g_{n-i-2}, \ldots, g_{0}\right), & 0<i<n \\
\left(\partial_{n} x, \partial_{n-1} g_{n-1}, \ldots, \partial_{1} g_{1}\right), & i=n,\end{cases} \\
& \quad \sigma_{i}\left(x, g_{n-1}, g_{n-2}, \ldots, g_{0}\right) \\
& \quad=\left(\sigma_{i} x, \sigma_{i-1} g_{n-1}, \ldots, \sigma_{0} g_{n-i}, e, g_{n-i-1}, g_{n-i-2}, \ldots, g_{0}\right)
\end{aligned}
$$

The space $[\bullet / G]$ is also denoted $\bar{W} G$, and is a model for the classifying space $B G$ of $G$. Note replacing $W G$ with any other fibrant cofibrant contractible $G$-space $E G$ will give the same properties. 
Lemma 1.7. If $G: s \mathcal{C}_{\Lambda} \rightarrow s \mathrm{Gp}$ is smooth, then $\bar{W} G$ is smooth.

Proof. For any surjection $A \rightarrow B$, we have $G(A) \rightarrow G(B)$ fibrant and surjective on $\pi_{0}$, which by Goerss and Jardine [3, Corollary V.6.9] implies that $\bar{W} G(A) \rightarrow \bar{W} G(B)$ is a fibration. If $A \rightarrow B$ is also acyclic, then everything is trivial by properties of $\bar{W}$ and $G$.

Remark 1.1. Observe that this is our first example of a quasi-smooth functor which is not a right Quillen functor for the simplicial model structure. The definitions of smoothness and quasi-smoothness were designed with $\bar{W} G$ in mind.

Lemma 1.8. If $X$ is quasi-smooth, then so is $[X / G] \rightarrow \bar{W} G$.

Proof. This follows from the observation that for any fibration (resp. trivial fibration) $Z \rightarrow Y$ of $G$-spaces, $[Z / G] \rightarrow[Y / G]$ is a fibration (resp. trivial fibration).

Corollary 1.1. If $X$ is quasi-smooth and $G$ smooth, then $[X / G]$ is quasismooth.

Proof. Consider the fibration $X \rightarrow[X / G] \rightarrow \bar{W} G$.

\subsection{Cohomology and obstructions}

Given a quasi-smooth morphism $\alpha: F \rightarrow G$ in $s c \mathrm{Sp}$, there exist $k$-vector spaces $H^{i}(F / G)$ for all $i \in \mathbb{Z}$.

By Pridham [14, Corollary 1.46], these have the property that for any simplicial $k$-vector space $V$ with finite-dimensional normalization,

$$
\pi_{m}\left(F(k \oplus V) \times_{G(k \oplus V)}\{0\}\right) \cong H^{-m}(F / G \otimes V),
$$

where $V^{2}=0$ and

$$
H^{i}(F / G \otimes V):=\bigoplus_{n \geq 0} H^{i+n}(F / G) \otimes \pi_{n}(V) .
$$

If $G=\bullet$ (the one-point set), we write $H^{j}(F):=H^{j}(F / \bullet)$.

We now have the following characterization of obstruction theory: 
Theorem 1.2. If $\alpha: F \rightarrow G$ in $s c \mathrm{Sp}$ is quasi-smooth, then for any small extension $e: I \rightarrow A \stackrel{f}{\rightarrow} B$ in $s \mathcal{C}_{\Lambda}$, there is a sequence of sets

$$
\pi_{0}(F A) \stackrel{f_{*}}{\longrightarrow} \pi_{0}\left(F B \times_{G B} G A\right) \stackrel{o_{e}}{\longrightarrow} H^{1}(F / G \otimes I)
$$

exact in the sense that the fibre of $o_{e}$ over 0 is the image of $f_{*}$. Moreover, there is a group action of $H^{0}(F / G \otimes I)$ on $\pi_{0}(F A)$ whose orbits are precisely the fibres of $f_{*}$.

For any $y \in F_{0} A$, with $x=f_{*} y$, the fibre of $F A \rightarrow F B \times_{G B} G A$ over $x$ is isomorphic to $\operatorname{ker}(\alpha: F I \rightarrow G I)$, and the sequence above extends to a long exact sequence

$$
\begin{aligned}
& \cdots \stackrel{f_{*}}{\longrightarrow} \pi_{n}\left(F B \times_{G B} G A, x\right) \stackrel{o_{e}}{\longrightarrow} H^{1-n}(F / G \otimes I) \stackrel{\partial_{e}}{\longrightarrow} \pi_{n-1}(F A, y) \stackrel{f_{*}}{\longrightarrow} \cdots \\
& \cdots \stackrel{f_{*}}{\longrightarrow} \pi_{1}\left(F B \times_{G B} G A, x\right) \stackrel{o_{e}}{\longrightarrow} H^{0}(F / G \otimes I) \stackrel{-* y}{\longrightarrow} \pi_{0}(F A) .
\end{aligned}
$$

Proof. See [14, Theorem 1.45].

Corollary 1.2. A map $\alpha: F \rightarrow G$ of quasi-smooth $F, G \in \operatorname{scSp}$ is a weak equivalence if and only if the maps $H^{j}(\alpha): H^{j}(F) \rightarrow H^{j}(G)$ are all isomorphisms.

Corollary 1.3. If $\alpha: F \rightarrow G$ is quasi-smooth in scSp, then $\alpha$ is smooth if and only if $H^{i}(F / G)=0$ for all $i>0$.

Proposition 1.3. Let $X, Y, Z: s \mathcal{C}_{\Lambda} \rightarrow \mathbb{S}$ be left-exact functors, with $X \stackrel{\alpha}{\rightarrow}$ $Y$ and $Y \stackrel{\beta}{\rightarrow} Z$ quasi-smooth. There is then a long exact sequence

$$
\begin{gathered}
\ldots \stackrel{\partial}{\rightarrow} H^{j}(X / Y) \rightarrow H^{j}(X / Z) \rightarrow H^{j}(Y / Z) \\
\stackrel{\partial}{\rightarrow} H^{j+1}(X / Y) \rightarrow H^{j+1}(X / Z) \rightarrow \ldots
\end{gathered}
$$

Proof. See [14, Proposition 1.61].

\subsection{Model structures}

Theorem 1.3. There is a simplicial model structure (called the geometric model structure) on scSp, for which the fibrations are quasi-smooth morphisms, and weak equivalences between quasi-smooth objects are those given in Definition 1.14.

Proof. This is [14, Theorem 2.14]. 
Thus the homotopy category $\mathrm{Ho}(s c \mathrm{Sp})$ is equivalent to the category of quasi-smooth objects in $s c \mathrm{Sp}$, localized at the weak equivalences of Definition 1.14.

Definition 1.16. Given any morphism $f: X \rightarrow Z$, we define $H^{n}(X / Z):=$ $H^{n}(\hat{X} / Z)$, for $X \stackrel{i}{\rightarrow} \hat{X} \stackrel{p}{\rightarrow} Z$ a factorization of $f$ with $i$ a geometric trivial cofibration, and $p$ a geometric fibration.

\subsubsection{Homotopy representability}

Definition 1.17. Define the category $\mathcal{S}$ to consist of functors $F: s \mathcal{C}_{\Lambda} \rightarrow \mathbb{S}$ satisfying the following conditions:

(A0) $F(k)$ is contractible.

(A1) For all small extensions $A \rightarrow B$ in $s \mathcal{C}_{\Lambda}$, and maps $C \rightarrow B$ in $s \mathcal{C}_{\Lambda}$, the map $F\left(A \times{ }_{B} C\right) \rightarrow F(A) \times{ }_{F(B)}^{h} F(C)$ is a weak equivalence, where $\times^{h}$ denotes homotopy fibre product.

(A2) For all acyclic small extensions $A \rightarrow B$ in $s \mathcal{C}_{\Lambda}$, the map $F(A) \rightarrow F(B)$ is a weak equivalence.

Say that a natural transformation $\eta: F \rightarrow G$ between such functors is a weak equivalence if the maps $F(A) \rightarrow G(A)$ are weak equivalences for all $A \in s \mathcal{C}_{\Lambda}$, and let $\operatorname{Ho}(\mathcal{S})$ be the category obtained by formally inverting all weak equivalences in $\mathcal{S}$.

Remark 1.4. We may apply the long exact sequence of homotopy to describe the homotopy groups of homotopy fibre products. If $f: X \rightarrow Z$, $g: Y \rightarrow Z$ in $\mathbb{S}$ and $P=X \times_{Z}^{h} Y$, the map $\theta: \pi_{0}(P) \rightarrow \pi_{0}(X) \times_{\pi_{0}(Z)} \pi_{0}(Y)$ is surjective. Moreover, $\pi_{1}(Z, *)$ acts transitively on the fibres of $\theta$ over $* \in \pi_{0} Z$.

Take $v \in \pi_{0}(P)$ over $*$. Then there is a connecting homomorphism $\partial$ : $\pi_{n}(Z, *) \rightarrow \pi_{n-1}(P, v)$ for all $n \geq 1$, giving a long exact sequence

$$
\ldots \stackrel{\partial}{\rightarrow} \pi_{n}(P, v) \rightarrow \pi_{n}(X, v) \times \pi_{n}(Y, v) \stackrel{f \cdot g^{-1}}{\longrightarrow} \pi_{n}(Z, *) \stackrel{\partial}{\rightarrow} \pi_{n-1}(P, v) \ldots
$$

Theorem 1.5. There is a canonical equivalence between the geometric homotopy category $\mathrm{Ho}(s c \mathrm{Sp})$ and the category $\operatorname{Ho}(\mathcal{S})$.

Proof. This is [14, Theorem 2.30]. 
1.6.2. Equivalent formulations If $k$ is a field of characteristic 0, then we may work with dg algebras rather than simplicial algebras.

Definition 1.18. Define $d g \mathcal{C}_{\Lambda}$ to be the category of Artinian local differential $\mathbb{N}_{0}$-graded graded-commutative $\Lambda$-algebras with residue field $k$.

Definition 1.19. Define a map $A \rightarrow B$ in $d g \mathcal{C}_{\Lambda}$ to be a small extension if it is surjective and the kernel $I$ satisfies $I \cdot \mathfrak{m}(A)=0$.

Definition 1.20. Define $s D G S p$ to be the category of left exact functors from $d g \mathcal{C}_{\Lambda}$ to $\mathbb{S}$.

Definition 1.21. Say a map $X \rightarrow Y$ in $s D G$ Sp is quasi-smooth if for all small extensions $f: A \rightarrow B$ in $d g \mathcal{C}_{\Lambda}$, the morphism

$$
X(A) \rightarrow Y(A) \times_{Y(B)} X(B)
$$

is a fibration in $\mathbb{S}$, which is moreover a trivial fibration if $f$ is acyclic.

Definition 1.22. We will say that a morphism $\alpha: F \rightarrow G$ of quasi-smooth objects of $s D G$ Sp is a weak equivalence if, for all $A \in s \mathcal{C}_{\Lambda}$, the maps $\pi_{i} F$ $(A) \rightarrow \pi_{i} G(A)$ are isomorphisms for all $i$.

Proposition 1.4. There is a model structure on sDGSp, for which the fibrations are quasi-smooth morphisms, and weak equivalences between quasismooth objects are those given in Definition 1.22.

Proof. This is [14, Proposition 4.12].

Most of the constructions from $s \mathcal{C}_{\Lambda}$ carry over to $d g \mathcal{C}_{\Lambda}$. However, there is no straightforward analogue of Definition 1.12.

Definition 1.23. Define the normalization functor $N: s \mathcal{C}_{\Lambda} \rightarrow d g \mathcal{C}_{\Lambda}$ by mapping $A$ to its associated normalized complex $N A$, equipped with the Eilenberg-Zilber shuffle product (as in [15]).

Definition 1.24. Define $\operatorname{Spf} N^{*}: s D G \operatorname{Sp} \rightarrow s c \operatorname{Sp}$ by mapping $X: d g \mathcal{C}_{\Lambda} \rightarrow$ $\mathbb{S}$ to the composition $X \circ N: s \mathcal{C}_{\Lambda} \rightarrow \mathbb{S}$. Note that this is well defined, since $N$ is left exact.

Theorem 1.6. Spf $N^{*}: s D G \mathrm{Sp} \rightarrow$ scSp is a right Quillen equivalence. 
Proof. This is [14, Theorem 4.18].

In particular, this means that $\operatorname{Spf} N^{*}$ maps quasi-smooth morphisms to quasi-smooth morphisms, and induces an equivalence $\mathbf{R S p f} N^{*}$ : Ho $(s D G \mathrm{Sp}) \rightarrow \mathrm{Ho}(s c \mathrm{Sp})$.

Now assume that $\Lambda=k$.

Theorem 1.7. $\operatorname{Ho}(s D G \mathrm{Sp})$ is equivalent to the category of $L_{\infty}$-algebras localized at tangent quasi-isomorphisms (as considered in [7]). This is also equivalent to the category of DGLAs (see Section 5.1) localized at quasiisomorphisms.

Proof. Combine Proposition 4.42 and Corollary 4.57 of [14].

\section{Monads and comonads}

\subsection{Algebras and coalgebras}

Definition 2.1. A monad (or triple) on a category $\mathcal{B}$ is a monoid in the category of endofunctors of $\mathcal{B}$ (with the monoidal structure given by composition of functors). A comonoid (or cotriple) is a comonoid in the category of endofunctors of $\mathcal{B}$.

The following is standard:

Lemma 2.1. Take an adjunction

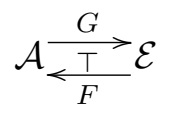

with unit $\eta: \mathrm{id} \rightarrow G F$ and co-unit $\varepsilon: F G \rightarrow \mathrm{id}$. Then $\top:=G F$ is a monad with unit $\eta$ and multiplication $\mu:=G \varepsilon F$, while $\perp:=F G$ is a comonad, with co-unit $\varepsilon$ and comultiplication $\Delta:=F \eta G$.

Definition 2.2. Given a monad $(\top, \mu, \eta)$ on a category $\mathcal{E}$, define the category $\mathcal{E}^{\top}$ of $\top$-algebras to have objects

$$
\top E \stackrel{\ominus}{\rightarrow} E,
$$

such that $\theta \circ \eta_{E}=$ id and $\theta \circ \top \theta=\theta \circ \mu_{E}$. 
A morphism

$$
g:\left(\top E_{1} \stackrel{\theta}{\rightarrow} E_{1}\right) \rightarrow\left(\top E_{2} \stackrel{\phi}{\rightarrow} E_{2}\right)
$$

of $\top$-algebras is a morphism $g: E_{1} \rightarrow E_{2}$ in $\mathcal{E}$ such that $\phi \circ \top g=g \circ \theta$.

We define the comparison functor $K: \mathcal{A} \rightarrow \mathcal{E}^{\top}$ by

$$
B \mapsto\left(U F U B \stackrel{U \varepsilon_{B}}{\longrightarrow} U B\right)
$$

on objects, and $K(g)=U(g)$ on morphisms.

Definition 2.3. The adjunction

$$
\mathcal{A}_{<\frac{U}{F}}^{\stackrel{U}{\longrightarrow}} \mathcal{E}
$$

is said to be monadic (or tripleable) if $K: \mathcal{A} \rightarrow \mathcal{E}^{\top}$ is an equivalence.

Examples 2.1. Intuitively, monadic adjunctions correspond to algebraic theories, such as the adjunction

$$
\operatorname{Ring} \frac{U}{\frac{T}{\mathbb{Z}[-]}} \text { Set }
$$

between rings and sets, $U$ being the forgetful functor. Other examples are $k$-algebras over $k$-vector spaces, or groups over sets.

Definition 2.4. Dually, given a comonad $(\perp, \Delta, \varepsilon)$ on a category $\mathcal{A}$, we define the category $\mathcal{A}_{\perp}$ of $\perp$-coalgebras by

$$
\left(\mathcal{A}_{\perp}\right)^{\mathrm{opp}}:=\left(\mathcal{A}^{\mathrm{opp}}\right)^{\perp \text { opp }}
$$

noting that $\perp^{\text {opp }}$ is a monad on the opposite category $\mathcal{A}^{\text {opp }}$. The adjunction of Lemma 2.1 is said to be comonadic (or cotripleable) if the adjunction on opposite categories is monadic.

Example 2.2. If $X$ is a topological space (or any site with enough points) and $X^{\prime}$ is the set of points of $X$, let $u: X^{\prime} \rightarrow X$ be the associated morphism. Then the adjunction $u^{-1} \dashv u_{*}$ on sheaves is comonadic, so the category of sheaves on $X$ is equivalent to $u^{-1} u_{*}$-coalgebras in the category of sheaves (or equivalently presheaves) on $X^{\prime}$.

A more prosaic example is that for any ring $A$, the category of $A$-coalgebras is comonadic over the category of $A$-modules. 


\subsection{Bialgebras}

As in [17, Section IV], take a category $\mathcal{B}$ equipped with both a monad $(\top, \mu, \eta)$ and a comonad $(\perp, \Delta, \gamma)$, together with a distributivity transformation $\lambda: \top \perp \Longrightarrow \perp \top$ for which the following diagrams commute:
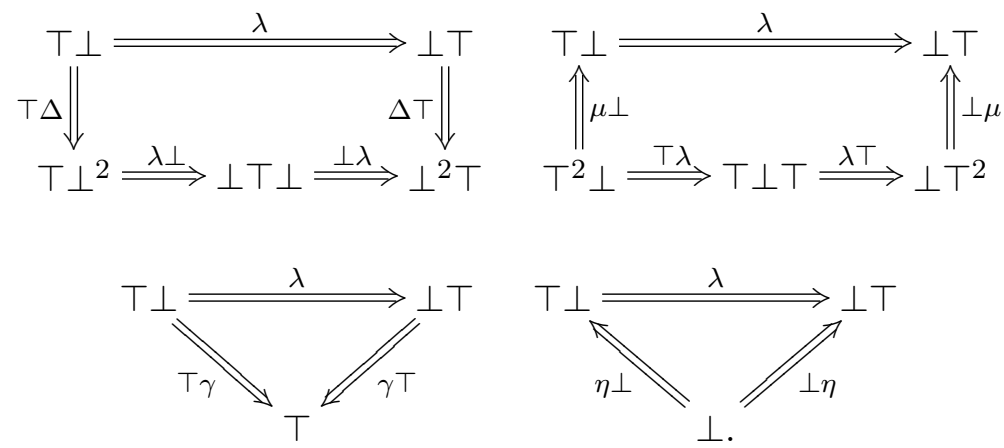

Definition 2.5. Given a distributive monad-comonad pair $(\top, \perp)$ on a category $\mathcal{B}$, define the category $\mathcal{B}_{\perp}^{\top}$ of bialgebras as follows. The objects of $\mathcal{B}_{\perp}^{\top}$ are triples $(\alpha, B, \beta)$ with $(\top B \stackrel{\alpha}{\rightarrow} B)$ an object of $\mathcal{B}^{\top}$ and $B \stackrel{\beta}{\rightarrow} \perp B$ an object of $\mathcal{B}_{\perp}$, such that the composition $(\beta \circ \alpha): \top B \rightarrow \perp B$ agrees with the composition

$$
\top B \stackrel{\top \beta}{\longrightarrow} \top \perp B \stackrel{\lambda}{\rightarrow} \perp \top B \stackrel{\perp \alpha}{\longrightarrow} \perp B .
$$

A morphism $f:(\alpha, B, \beta) \rightarrow\left(\alpha^{\prime}, B^{\prime}, \beta^{\prime}\right)$ is a morphism $f: B \rightarrow B^{\prime}$ in $\mathcal{B}$ such that $\alpha^{\prime} \circ \top f=f \circ \alpha$ and $\beta^{\prime} \circ f=\perp f \circ \beta$.

To understand how the data $(\top, \perp, \eta, \mu, \gamma, \Delta, \lambda)$ above occur naturally, note that by Van Osdol [17, Section IV] or [11, Section 2], these data are equivalent to a diagram

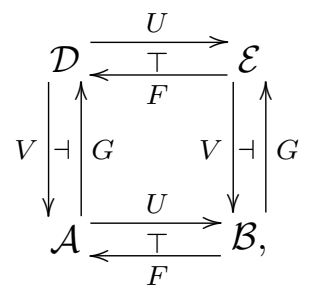

with $F \dashv U$ monadic, $G \vdash V$ comonadic and $U, V$ commuting with everything (although $G$ and $F$ need not commute). The associated monad is $\top=U F$, and the comonad $\perp=V G$. Distributivity ensures that $\mathcal{D} \simeq \mathcal{E}^{\top} \simeq$ $\left(\mathcal{B}_{\perp}\right)^{\top}$ and $\mathcal{D} \simeq \mathcal{A}_{\perp} \simeq\left(\mathcal{B}^{\top}\right)_{\perp}$. In other words, $\mathcal{D} \simeq \mathcal{B}_{\perp}^{\top}$. 
Example 2.3. If $X$ is a topological space (or any site with enough points) and $X^{\prime}$ is the set of points of $X$, let $\mathcal{D}$ be the category of sheaves of rings on $X$. If $\mathcal{B}$ is the category of sheaves (or equivalently presheaves) of sets on $X^{\prime}$, then the description above characterizes $\mathcal{D}$ as a category of bialgebras over $\mathcal{B}$, with the comonad being $u^{-1} u_{*}$ for $u: X^{\prime} \rightarrow X$, and the monad being the free polynomial functor.

\section{Constructing SDCs}

Recall the definition of an SDC:

Definition 3.1. A simplicial deformation complex $E^{\bullet}$ consists of smooth left-exact functors $E^{n}: \mathcal{C}_{\Lambda} \rightarrow$ Set for each $n \geq 0$, together with maps

$$
\begin{array}{ll}
\partial^{i}: E^{n} \rightarrow E^{n+1}, & 1 \leq i \leq n, \\
\sigma^{i}: E^{n} \rightarrow E^{n-1}, & 0 \leq i<n,
\end{array}
$$

an associative product $*: E^{m} \times E^{n} \rightarrow E^{m+n}$, with identity $1: \bullet \rightarrow E^{0}$, where $\bullet$ is the constant functor $\bullet(A)=\bullet$ (the one-point set) on $\mathcal{C}_{\Lambda}$, such that:

(1) $\partial^{j} \partial^{i}=\partial^{i} \partial^{j-1}, \quad i<j$.

(2) $\sigma^{j} \sigma^{i}=\sigma^{i} \sigma^{j+1}, \quad i \leq j$.

(3) $\sigma^{j} \partial^{i}= \begin{cases}\partial^{i} \sigma^{j-1}, & i<j, \\ \mathrm{id}, & i=j, i=j+1, \\ \partial^{i-1} \sigma^{j}, & i>j+1 .\end{cases}$

(4) $\partial^{i}(e) * f=\partial^{i}(e * f)$.

(5) $e * \partial^{i}(f)=\partial^{i+m}(e * f)$, for $e \in E^{m}$.

(6) $\sigma^{i}(e) * f=\sigma^{i}(e * f)$.

(7) $e * \sigma^{i}(f)=\sigma^{i+m}(e * f)$, for $e \in E^{m}$.

From the viewpoint of homotopical algebra, there is a more natural way of characterizing the smoothness criterion for $E^{\bullet}$. Analogously to [3, Lemma VII.4.9], we define matching objects by $M^{-1} E:=\bullet, M^{0} E:=E^{0}$ and for $n>0$

$$
M^{n} E=\left\{\left(e_{0}, e_{1}, \ldots, e_{n}\right) \in\left(E^{n}\right)^{n+1} \mid \sigma^{i} e_{j}=\sigma^{j-1} e_{i} \forall i<j\right\}
$$


Proposition 3.1. The canonical maps $\underline{\sigma}: E^{n+1} \rightarrow M^{n} E$, given by $e \mapsto$ $\left(\sigma^{0} e, \sigma^{1} e, \ldots, \sigma^{n} e\right)$, are all smooth, for $n \geq 0$.

Proof. Since $E^{n}$ is smooth, by the Standard Smoothness Criterion (e.g., [8, Proposition 2.17]) it suffices to show that this is surjective on tangent spaces. The tangent space of $M^{n} E$ consists of $(n+1)$-tuples $\gamma_{i} \in C^{n}(E)$ satisfying $\sigma^{i} \gamma_{j}=\sigma^{j-1} \gamma_{i}$, for $i<j$. For any cosimplicial complex $C^{\bullet}$, there is a decomposition of the associated cochain complex as $C^{n}=N_{c}^{n}(C) \oplus$ $D^{n}(C)$, where $N_{c}^{n}(C)=\cap_{i=0}^{n-1} \operatorname{ker} \sigma^{i}$, and $D^{n}(C)=\sum_{i=1}^{n} \partial^{i} C^{n-1}$. Moreover $\underline{\sigma}: D^{n} \rightarrow M^{n-1} C$ is an isomorphism, giving the required surjectivity.

Definition 3.2. Given an SDC E, recall from [11] that the Maurer-Cartan functor $\mathrm{MC}_{E}: \mathcal{C}_{\Lambda} \rightarrow$ Set is defined by

$$
\operatorname{MC}_{E}(A)=\left\{\omega \in E^{1}(A): \omega * \omega=\partial^{1}(\omega)\right\}
$$

The group $E^{0}(A)$ acts on this by conjugation, and we define $\mathfrak{D e f}_{E}(A)$ to be the groupoid with objects $\mathrm{MC}_{E}(A)$ and morphisms given by $E^{0}(A)$ via this action. We say that an SDC governs a deformation problem if $\mathfrak{D} \mathfrak{e} \mathfrak{f}_{E}$ is equivalent to the associated deformation functor.

Definition 3.3. Recall that $C^{\bullet}(E)$ denotes the tangent space of $E^{\bullet}$, i.e., $C^{n}(E)=E^{n}(k[\epsilon])$ for $\epsilon^{2}=0$. This has the natural structure of a cosimplicial complex, by Pridham [11], and we set $H^{i}(E):=H^{i}\left(C^{\bullet}(E)\right)$.

\subsection{SDCs from bialgebraic structures}

Definition 3.4. Recall from [13] that $\Delta_{* *}$ is defined to be the subcategory of the ordinal number category $\Delta$ containing only those non-decreasing morphisms $f: \mathbf{m} \rightarrow \mathbf{n}$ with $f(0)=0, f(m)=n$. We define a monoidal structure on this category by setting $\mathbf{m} \otimes \mathbf{n}=\mathbf{m}+\mathbf{n}$, with

$$
(f \otimes g)(i)= \begin{cases}f(i), & i \leq m \\ g(i-m)+n, & i \geq m\end{cases}
$$

for $f: \mathbf{m} \rightarrow \mathbf{n}$.

Definition 3.5. As in [13], define monoidal structures on $\operatorname{Set}^{\Delta_{* *}}$ and $\mathbb{S}^{\Delta_{* *}}$ by setting

$$
(X \otimes Y)^{n}:=\coprod_{a+b=n} X^{a} \otimes Y^{b},
$$


with operations given by

$$
\begin{array}{r}
\partial^{i}(x \otimes y)= \begin{cases}\left(\partial^{i} x\right) \otimes y, & i \leq a, \\
x \otimes\left(\partial^{i-a} y\right), & i>a,\end{cases} \\
\sigma^{i}(x \otimes y)= \begin{cases}\left(\sigma^{i} x\right) \otimes y, & i<a, \\
x \otimes\left(\sigma^{i-a} y\right), & i \geq a .\end{cases}
\end{array}
$$

The identity $I$ is given by $I^{0}=\bullet$ and $I^{n}=\emptyset$ for $n>0$.

Note that an SDC over $\Lambda$ is a smooth left-exact functor from $\mathcal{C}_{\Lambda}$ to the category of monoids in $\operatorname{Set}^{\Delta_{* *}}$.

Assume that we have a diagram

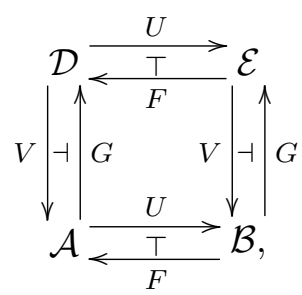

of homogeneous (i.e., preserving fibre products, but not the final object) functors from $\mathcal{C}_{\Lambda}$ to Cat as in [11, Section 2] (i.e., $\mathcal{B}$ has uniformly trivial deformation theory, with the diagram satisfying the conditions of Section 2.2). Recall that we write $\top_{\mathrm{h}}=U F, \perp_{\mathrm{h}}=F U, \perp_{\mathrm{v}}=V G$ and $\mathrm{\top}_{\mathrm{v}}=G V$.

Proposition 3.2. For the diagram above and $A \in \mathcal{C}_{\Lambda}, \mathcal{B}(A)$ has the structure of category enriched in $\mathrm{Set}^{\Delta_{* *}}$, with

$$
\mathcal{H o m}_{\mathcal{B}}\left(B, B^{\prime}\right)^{n}=\operatorname{Hom}_{\mathcal{B}}\left(\top_{\mathrm{h}}^{n} B, \perp_{\mathrm{v}}^{n} B^{\prime}\right)
$$

Proof. See [13, Proposition 2.12].

Examples 3.1. (1) If $X$ is a topological space (or any site with enough points) and $X^{\prime}$ is the set of points of $X$, let $\mathcal{D}(A)$ be the category of sheaves of flat $A$-algebras on $X^{\prime}$. If $\mathcal{B}$ is the category of sheaves (or equivalently presheaves) of flat $A$-modules on $X^{\prime}$, then the description above characterizes $\mathcal{D}$ as $\mathcal{B}_{\perp_{\mathrm{v}}}^{\top_{\mathrm{h}}}$, with $\perp_{\mathrm{v}}=u^{-1} u_{*}$ for $u: X^{\prime} \rightarrow X$, and $\top_{\mathrm{h}}$ being the free $A$-algebra functor for module. This example arises when considering deformations of a scheme $X$ in [11, Section 3.2], since deformations of $X$ are equivalent to deformations of the sheaf $\mathscr{O}_{X}$ of algebras. 
(2) Another important example (considered in [11, Section 3.1]) is when $\mathcal{D}(A)$ is the category of flat Hopf algebras over $A$, with $\mathcal{A}(A), \mathcal{E}(A)$ and $\mathcal{B}(A)$ the categories of flat algebras, coalgebras and modules, respectively.

(3) In order to make the first example functorial, we could let $\mathcal{B}$ be the category of pairs $\left(\left\{M_{x}\right\}_{x \in X}, X\right)$, for $X$ a topological space and $\left\{M_{x}\right\}_{x \in X}$ a presheaf of flat $A$-modules on $X^{\prime}$, with a morphism $f^{\sharp}$ : $\left(\left\{N_{y}\right\}_{y \in Y}, Y\right) \rightarrow\left(\left\{M_{x}\right\}_{x \in X}, X\right)$ given by a map $f: X \rightarrow Y$ of topological spaces, together with maps $f_{x}^{\sharp}: N_{f(x)} \rightarrow M_{x}$ for all $x \in X$.

We may define $\perp_{\mathrm{v}}$ and $T_{\mathrm{h}}$ as before, and then $\mathcal{B}_{\perp_{\mathrm{v}}}^{T_{\mathrm{h}}}$ will be the category of pairs $\left(\mathscr{O}_{X}, X\right)$, where $X$ is a topological space and $\mathscr{O}_{X}$ a sheaf of flat $A$-algebras on $X$.

\subsection{SDCs from diagrams}

Definition 3.6. Given a morphism $f: D \rightarrow D^{\prime}$ in $\mathcal{D}(k)$, choose lifts $B, B^{\prime} \in$ $\mathcal{B}(\Lambda)$ of $U V D, U V D^{\prime} \in \mathcal{B}(k)$ (which exist since the deformation theory of $\mathcal{B}$ is uniformly trivial). Then define

$$
E_{\mathcal{D} / \mathcal{B}}^{n}(f):=\mathscr{H}_{0 m^{n}}\left(B, B^{\prime}\right)_{U V\left(\alpha_{D^{\prime}}^{n} \circ f \circ \varepsilon_{D}^{n}\right)}: \mathcal{C}_{\Lambda} \rightarrow \text { Set }
$$

where $\alpha: 1 \rightarrow \top_{\mathrm{v}}$ and $\varepsilon: \perp_{\mathrm{h}} \rightarrow 1$ are the unit and counit of the respective adjunctions.

Write $E_{\mathcal{D} / \mathcal{B}}^{*}(D):=E_{\mathcal{D} / \mathcal{B}}^{*}\left(\mathrm{id}_{D}\right)$. Note that uniform triviality of $\mathcal{B}$ ensures that these constructions are independent of the choices of lift, since any other choice is isomorphic.

Lemma 3.1. $E_{\mathcal{D} / \mathcal{B}}(f)(A)$ has the natural structure of a cosimplicial complex.

For every pair of composable morphisms $f, g$ in $\mathcal{D}(k)$ between such objects, there is a product

$$
E_{\mathcal{D} / \mathcal{B}}(f)(A) \otimes E_{\mathcal{D} / \mathcal{B}}(g)(A) \rightarrow E_{\mathcal{D} / \mathcal{B}}(f \circ g)(A)
$$

in Set $^{\Delta_{* *}}$, functorial in $A$. 
Proof. It follows from Proposition 3.2 that $E_{\mathcal{D} / \mathcal{B}}(f)(A) \in S t^{\Delta_{* *}}$, with operations

$$
\begin{aligned}
& \partial^{i}(x)=\perp_{\mathrm{v}}^{i-1} V \alpha_{G \perp_{\mathrm{v}}^{n-i} B} \circ x \circ \top_{\mathrm{h}}^{i-1} U \varepsilon_{F \top_{\mathrm{h}}^{n-i} B}, \quad 0<i \leq n, \\
& \sigma^{i}(x)=\perp_{\mathrm{v}}^{i} \gamma_{\perp_{\mathrm{v}}^{n-i-1} B} \circ x \circ \top_{\mathrm{h}}^{i} \eta_{\top_{\mathrm{h}}^{n-i-1} B}, \quad 0 \leq i<n,
\end{aligned}
$$

for $\eta: 1 \rightarrow \top_{\mathrm{h}}$ and $\gamma: \perp_{\mathrm{v}} \rightarrow 1$ the respective unit and co-unit. The multiplication also follows from Proposition 3.2.

The canonical object of $\operatorname{MC}\left(E_{\mathcal{D} / \mathcal{B}}\left(\operatorname{id}_{D}\right)(\Lambda)\right)$ corresponding to $D$ gives an element $\omega_{D} \in E_{\mathcal{D} / \mathcal{B}}\left(\operatorname{id}_{D}\right)(\Lambda)^{1}$ and we then enhance the structure above to give a cosimplicial structure by setting

$$
\partial^{0} x:=\omega_{D^{\prime}} * x, \quad \partial^{n+1} x=x * \omega_{D},
$$

for $x \in E_{\mathcal{D} / \mathcal{B}}(f)(A)^{n}$.

Definition 3.7. Given an SDC $E$, and a simplicial set $X$, define an SDC $E^{X}$ by

$$
\left(E^{X}\right)^{n}=\left(E^{n}\right)^{X_{n}} .
$$

For $x \in X_{n+1}, y \in Y_{n+1}, z \in X_{m+n}, 1 \leq i \leq n, 0 \leq j<n, e \in\left(E^{X}\right)^{n}$ and $f \in\left(E^{X}\right)^{m}$, we define the operations by

$$
\begin{aligned}
\partial^{i}(e)(x) & :=\partial^{i}\left(e\left(\partial_{i} x\right)\right) \\
\sigma^{j}(e)(y) & :=\sigma^{j}\left(e\left(\sigma_{i} y\right)\right) \\
(f * e)(z) & :=f\left(\left(\partial_{m+1}\right)^{n} z\right) * e\left(\left(\partial_{0}\right)^{m} z\right) .
\end{aligned}
$$

Definition 3.8. Let $C_{\mathcal{D} / \mathcal{B}}^{\bullet}(f)$ be the the tangent space of $E_{\mathcal{D} / \mathcal{B}}(f)$. This is a vector space over $k$, and we define

$$
\operatorname{Ext}_{\mathcal{D} / \mathcal{B}}^{*}(f):=H^{*}\left(C_{\mathcal{D} / \mathcal{B}}^{\bullet}(f)\right)
$$

this construction is closely related to Van Osdol's bicohomology [17].

Lemma 3.2. If $X$ is a finite simplicial set, then

$$
H^{n}\left(E^{X}\right) \cong \bigoplus_{i+j=n} H^{i}(E) \otimes H^{j}(X, k) .
$$

Proof. Since $X$ is finite, $C^{\bullet}\left(E^{X}\right) \cong C^{\bullet}(E) \otimes k^{X}$, and the result now follows from the Künneth formula. 
Definition 3.9. Given a small category $\mathbb{I}$ and an $\mathbb{I}$-diagram $\mathbb{D}: \mathbb{I} \rightarrow \mathcal{D}(k)$, define the $\operatorname{SDC} E_{\mathcal{D} / \mathcal{B}}^{\bullet}(\mathbb{D})$ by

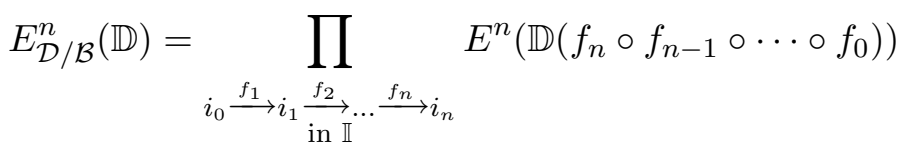

$$
\begin{aligned}
& =\prod_{x \in B \mathbb{I}_{n}} E^{n}\left(\mathbb{D}\left(\partial_{1}{ }^{n-1} x\right)\right),
\end{aligned}
$$

where $B \mathbb{I}$ is the nerve of $\mathbb{I}\left(\right.$ so $\left.B \mathbb{I}_{0}=\mathrm{Ob}(\mathbb{I}), B \mathbb{I}_{1}=\operatorname{Mor}(\mathbb{I})\right)$, and $\partial_{1}^{-1}:=\sigma_{0}$.

We define the operations by the formulae of Definition 3.7.

Theorem 3.2. The $S D C E_{\mathcal{D} / \mathcal{B}}^{\bullet}(\mathbb{D})$ governs deformations of the diagram $\mathbb{D}: \mathbb{I} \rightarrow \mathcal{D}(k)$.

Proof. This follows immediately from [13, Lemma 1.36], which characterizes objects of $\mathfrak{D} \mathfrak{e} \mathfrak{f}_{E}$ as diagrams from $\mathbb{I}$ to a category equivalent to $\mathcal{D}(A)$.

Lemma 3.3. Given a diagram $\mathbb{D}: \mathbb{I} \rightarrow \mathcal{D}(k)$, the cohomology groups $H^{*}$ $\left(E_{\mathcal{D} / \mathcal{B}}^{\bullet}(\mathbb{D})\right)$ are given by hypercohomology of the bicomplex

$$
\begin{aligned}
& \prod_{i \in \mathrm{Ob} \mathbb{I}} C_{\mathcal{D} / \mathcal{B}}^{\bullet}(\mathbb{D}(i)) \stackrel{f_{*}-f^{*}}{\longrightarrow} \ldots \rightarrow \\
& \left.\prod \quad C_{\mathcal{D} / \mathcal{B}}^{\bullet}\left(\mathbb{D}\left(f_{n} \circ f_{n-1} \circ \ldots f_{0}\right)\right)\right) \rightarrow \ldots \\
& i_{0} \stackrel{f_{1}}{\longrightarrow} i_{1} \underset{\text { in } N_{n} \mathbb{I}}{\stackrel{f_{2}}{\longrightarrow} \ldots} \stackrel{f_{n}}{\longrightarrow} i_{n}
\end{aligned}
$$

where $N_{n} \mathbb{I} \subset B_{n} \mathbb{I}$ consists of non-degenerate simplices, or equivalently strings of non-identity morphisms.

Proof. Since $C^{\bullet}\left(E_{\mathcal{D} / \mathcal{B}}^{\bullet}(\mathbb{D})\right)$ is the diagonal of the bicosimplicial complex

$$
\begin{aligned}
& {[m, n] \mapsto \quad \prod \quad C_{\mathcal{D} / \mathcal{B}}^{m}\left(\mathbb{D}\left(f_{n} \circ f_{n-1} \circ \ldots f_{0}\right)\right),} \\
& i_{0} \stackrel{f_{1}}{\longrightarrow} i_{1} \underset{\text { in II }}{\stackrel{f_{2}}{\longrightarrow}} \stackrel{f_{n}}{\longrightarrow} i_{n}
\end{aligned}
$$

the Eilenberg-Zilber theorem implies that it is homotopy equivalent to the total complex of the associated binormalized complex. The vertical normalisation is just given by replacing $B_{n} \mathbb{I}$ with $N_{n} \mathbb{I}$. 
Example 3.3. If we define $\mathcal{D}$ and $\mathcal{B}$ as in Example 3.1.3, then the category of flat schemes over $A$ is a full subcategory of $\mathcal{D}(A)$, closed under deformations. Therefore, Theorem 3.2 constructs an SDC governing deformations of a diagram of schemes.

For a morphism $f:\left(X, \mathscr{O}_{X}\right) \rightarrow\left(Y, \mathscr{O}_{Y}\right)$ in $\mathcal{D}(k)^{\mathrm{opp}}$, the reasoning of $[11$, Section 3.2 adapts to show that

$$
\operatorname{Ext}_{\mathcal{D} / \mathcal{B}}^{*}(f)=\mathbb{E x t}_{\mathscr{O}_{Y}}^{*}\left(\mathbf{L}_{\bullet}^{Y / k}, \mathbf{R} f_{*} \mathscr{O}_{X}\right)=\mathbb{E x t}_{\mathscr{O}_{X}}^{*}\left(f^{*} \mathbf{L}_{\bullet}^{Y / k}, \mathscr{O}_{X}\right),
$$

where $\mathbf{L}_{\bullet}^{Y / k}$ is the cotangent complex of [6].

\subsection{Constrained deformations}

We now consider a generalization of Section 3.2, by taking a small diagram

$$
\mathbb{D}: \mathbb{I} \rightarrow \mathcal{D}(k)
$$

a subcategory $\mathbb{J} \subset \mathbb{I}$, and $\left.\widetilde{\mathbb{D}}\right|_{\mathbb{J}}: \mathbb{J} \rightarrow \mathcal{D}(\Lambda)$ lifting $\left.\mathbb{D}\right|_{\mathbb{J}}$. We wish to describe deformations of $\mathbb{D}$ which agree with $\widetilde{\left.\mathbb{D}\right|_{\mathbb{J}}}$ on $\mathbb{J}$. Note that when $\mathbb{I}=(0 \rightarrow 1)$ and $\mathbb{J}=\{1\}$, this is the type of problem considered in $[2,16]$.

Proposition 3.3. Given a $\mathbb{I}$-diagram $\mathbb{D}: \mathbb{I} \rightarrow \mathcal{D}(k)$, with $\widetilde{\left.\mathbb{D}\right|_{\mathbb{J}}}$ as above, the groupoid of deformations of $\mathbb{D}$ fixing $\widetilde{\left.\mathbb{D}\right|_{\mathbb{J}}}$ is governed by the $S D C$ :

$$
E_{\mathcal{D} / \mathcal{B}}^{\bullet}(\mathbb{D}) \times_{E_{\mathcal{D} / \mathcal{B}}^{\bullet}\left(\left.\mathbb{D}\right|_{J}\right)} \bullet,
$$

where $\bullet \rightarrow E_{\mathcal{D} / \mathcal{B}}^{\bullet}\left(\left.\mathbb{D}\right|_{\mathbb{J}}\right)$ is defined by the object of $\mathrm{MC}\left(E_{\mathcal{D} / \mathcal{B}}^{\bullet}\left(\left.\mathbb{D}\right|_{\mathbb{J}}\right)\right)$ corresponding to $\left.\widetilde{\mathbb{D}}\right|_{\mathbb{J}}$.

Proof. By Theorem 3.2, it suffices to show that $\mathfrak{D e f}\left(E_{\mathcal{D} / \mathcal{B}}^{\bullet}(\mathbb{D}) \times_{E_{\mathcal{D} / \mathcal{B}}^{\bullet}}\left(\left.\mathbb{D}\right|_{\mathbb{J}}\right) \bullet\right)$ is equivalent to the 2-fibre product

$$
\mathfrak{D} \mathfrak{e f}\left(E_{\mathcal{D} / \mathcal{B}}^{\bullet}(\mathbb{D})\right) \times_{\mathfrak{D} \mathfrak{e}\left(E^{\bullet}\left(\left.\mathbb{D}\right|_{\mathbb{J}}\right)\right)}^{h}\left(\left\{\widetilde{\left.\mathbb{D}\right|_{\mathbb{J}}}\right\}, \mathrm{id}\right) .
$$

We know that the functor MC preserves inverse limits, so

$$
\operatorname{MC}\left(E^{\bullet}(\mathbb{D}) \times_{E^{\bullet}\left(\left.\mathbb{D}\right|_{J}\right)}\left\{\widetilde{\left.\mathbb{D}\right|_{\mathbb{J}}}\right\}\right)=\operatorname{MC}\left(E_{\mathcal{D} / \mathcal{B}}^{\bullet}(\mathbb{D})\right) \times_{\operatorname{MC}\left(E^{\bullet}\left(\left.\mathbb{D}\right|_{\mathbb{J}}\right)\right)}\left\{\widetilde{\left.\mathbb{D}\right|_{\mathbb{J}}}\right\} .
$$

Since $E^{0}(\mathbb{D})(A) \rightarrow E^{0}\left(\left.\mathbb{D}\right|_{\mathbb{J}}\right)(A)$ is also surjective (by smoothness), we see that

$$
\mathfrak{D e f}\left(E^{\bullet}(\mathbb{D}) \times_{E^{\bullet}\left(\left.\mathbb{D}\right|_{\mathbb{J}}\right)}\left\{\widetilde{\left.\mathbb{D}\right|_{\mathbb{J}}}\right\}\right) \simeq \mathfrak{D} \mathfrak{e f}\left(E_{\mathcal{D} / \mathcal{B}}^{\bullet}(\mathbb{D})\right) \times_{\mathfrak{D} \mathfrak{e f}\left(E^{\bullet}\left(\left.\mathbb{D}\right|_{\mathbb{J}}\right)\right)}^{h}\left(\left\{\widetilde{\left.\mathbb{D}\right|_{\mathbb{J}}}\right\}, \mathrm{id}\right) .
$$


Example 3.4. Given a morphism $f: X \rightarrow Y$ of schemes over $k$, and a flat formal deformation $\mathfrak{Y}$ of $Y$ over $\Lambda$, we may consider deformations of $X$ over $\mathfrak{Y}$, or equivalently deformations of this diagram fixing $\mathfrak{Y}$. Define the diagram $\mathbb{D}$ to be $f^{\sharp}:\left(\mathscr{O}_{Y}, Y\right) \rightarrow\left(\mathscr{O}_{X}, X\right)$ in the category $\mathcal{D}(k)$ of Example 3.1.3, and let $\widetilde{\left.\mathbb{D}\right|_{\mathbb{J}}}$ be the object $\left(\mathscr{O}_{\mathfrak{Y}}, \mathfrak{Y}\right)$ of $\mathcal{D}(\Lambda)$. Proposition 3.3 then gives an SDC

$$
E:=E_{\mathcal{D} / \mathcal{B}}^{\bullet}(\mathbb{D}) \times_{E_{\mathcal{D} / \mathcal{B}}\left(\left.\mathbb{D}\right|_{J}\right)} \bullet
$$

governing this problem.

Lemma 3.3 implies that the tangent complex $C^{\bullet}(E)$ is the mapping cone of $C_{\mathcal{D} / \mathcal{B}}^{\bullet}\left(\mathscr{O}_{X}, X\right) \rightarrow C_{\mathcal{D} / \mathcal{B}}^{\bullet}\left(f^{\sharp}\right)$, so by Example 3.3 , the cohomology of this SDC is given by

$$
H^{*}(E) \cong \mathbb{E x t}_{\mathscr{O}_{X}}^{*}\left(\operatorname{cone}\left(f^{*} \mathbf{L}_{\bullet}^{Y / k} \rightarrow \mathbf{L}_{\bullet}^{X / k}\right), \mathscr{O}_{X}\right) \cong \mathbb{E x t}_{\mathscr{O}_{X}}^{*}\left(\mathbf{L}_{\bullet}^{X / Y}, \mathscr{O}_{X}\right) .
$$

Example 3.5. We could go further, and let $\mathbb{E}$ be a diagram $Z \stackrel{g}{\rightarrow} X \stackrel{f}{\rightarrow} Y$ over $k$, with a fixed formal deformation $\widetilde{g f}: \mathfrak{Z} \rightarrow \mathfrak{Y}$ of $f g$ over $\Lambda$. Governing this deformation problem, we get another SDC:

$$
F:=E_{\mathcal{D} / \mathcal{B}}^{\bullet}(Z \stackrel{g}{\rightarrow} X \stackrel{f}{\rightarrow} Y) \times_{E_{\mathcal{D} / \mathcal{B}}^{\bullet}(Z \stackrel{g f}{\longrightarrow} Y)}\{\mathfrak{Z} \stackrel{\widetilde{g f}}{\longrightarrow} \mathfrak{Y}\} .
$$

Now, $C^{\bullet}(F)=\operatorname{ker}\left(C^{\bullet}\left(E_{\mathcal{D} / \mathcal{B}}(\mathbb{E})\right) \rightarrow C^{\bullet}\left(E_{\mathcal{D} / \mathcal{B}}\left(\left.\mathbb{E}\right|_{\mathbb{J}}\right)\right)\right)$, and Lemma 3.3 implies that $C^{\bullet}\left(E_{\mathcal{D} / \mathcal{B}}(\mathbb{E})\right)$ is homotopy equivalent to the total complex of

$$
\begin{aligned}
& C_{\mathcal{D} / \mathcal{B}}^{\bullet}(Z) \times C_{\mathcal{D} / \mathcal{B}}^{\bullet}(X) \times C_{\mathcal{D} / \mathcal{B}}^{\bullet}(Y) \rightarrow C_{\mathcal{D} / \mathcal{B}}^{\bullet}(g) \times C_{\mathcal{D} / \mathcal{B}}^{\bullet}(f g) \\
& \quad \times C_{\mathcal{D} / \mathcal{B}}^{\bullet}(f) \rightarrow C_{\mathcal{D} / \mathcal{B}}^{\bullet}(f g),
\end{aligned}
$$

while $C^{\bullet}\left(E_{\mathcal{D} / \mathcal{B}}\left(\left.\mathbb{E}\right|_{\mathbb{J}}\right)\right)$ is homotopy equivalent to the total complex of

$$
C_{\mathcal{D} / \mathcal{B}}^{\bullet}(Z) \times C_{\mathcal{D} / \mathcal{B}}^{\bullet}(Y) \rightarrow C_{\mathcal{D} / \mathcal{B}}^{\bullet}(f g),
$$

so $C^{\bullet}(F)$ is homotopy equivalent to the total complex of

$$
C_{\mathcal{D} / \mathcal{B}}^{\bullet}(X) \rightarrow C_{\mathcal{D} / \mathcal{B}}^{\bullet}(g) \times C_{\mathcal{D} / \mathcal{B}}^{\bullet}(f) \rightarrow C_{\mathcal{D} / \mathcal{B}}^{\bullet}(f g) .
$$

By Example 3.3,

$$
\begin{aligned}
& H^{*} \operatorname{ker}\left(C_{\mathcal{D} / \mathcal{B}}^{\bullet}(X) \rightarrow C_{\mathcal{D} / \mathcal{B}}^{\bullet}(f)\right) \cong \mathbb{E x t}_{\mathscr{O}_{X}}^{*}\left(\mathbf{L}_{\bullet}^{X / Y}, \mathscr{O}_{X}\right), \\
& H^{*} \operatorname{ker}\left(C_{\mathcal{D} / \mathcal{B}}^{\bullet}(g) \rightarrow C_{\mathcal{D} / \mathcal{B}}^{\bullet}(f g)\right) \cong \mathbb{E x t}_{\mathscr{O}_{X}}^{*}\left(\mathbf{L}_{\bullet}^{X / Y}, \mathbf{R} g_{*} \mathscr{O}_{Z}\right),
\end{aligned}
$$


and these isomorphisms combine to give

$$
H^{*}(F) \cong \mathbb{E x t}_{\mathscr{O}_{X}}^{*}\left(\mathbf{L}_{\bullet}^{X / Y}, \operatorname{cone}\left(\mathscr{O}_{X} \rightarrow \mathbf{R} g_{*} \mathscr{O}_{Z}\right)[-1]\right)
$$

Note that this more accurately captures the higher structure than the SDC of [11, Section 3.3], whose cohomology had $g_{*} \mathscr{O}_{Z}$ in place of $\mathbf{R} g_{*} \mathscr{O}_{Z}$ above.

\section{Extended deformation functors from SDCs}

Given an SDC $E$, the aim of this section is to extend the classical deformation groupoid $\mathfrak{D e f}_{E}: \mathcal{C}_{\Lambda} \rightarrow \operatorname{Grpd}$ of [11] from $\mathcal{C}_{\Lambda}$ to the whole of $s \mathcal{C}_{\Lambda}$. Groupoids turn out to be too restrictive for our purposes, so we will define a simplicial set-valued functor $\operatorname{Def}_{E}: s \mathcal{C}_{\Lambda} \rightarrow \mathbb{S}$ extending the classifying space $B \mathfrak{D e f} \mathfrak{f}_{E}$ of the deformation groupoid.

For a monad $T$, the obvious extension of the functor describing deformations of a $T$-algebra is the functor of deformations of a strong homotopy $T$-algebra. Strong homotopy algebras were defined by Lada in [1] to characterize the structures arising on deformation retracts of $T$-algebras in topological spaces, but the description works over any simplicial category. This motivates the following definition:

Definition 4.1. Given an SDC $E$, define the Maurer-Cartan functor $\mathrm{MC}_{E}$ : $s \mathcal{C}_{\Lambda} \rightarrow$ Set by

$$
\mathrm{MC}_{E}(A) \subset \prod_{n \geq 0} E^{n+1}\left(A^{I^{n}}\right),
$$

consisting of those $\underline{\omega}$ satisfying:

$$
\begin{aligned}
\omega_{m}\left(s_{1}, \ldots, s_{m}\right) * \omega_{n}\left(t_{1}, \ldots, t_{n}\right) & =\omega_{m+n+1}\left(s_{1}, \ldots, s_{m}, 0, t_{1}, \ldots, t_{n}\right), \\
\partial^{i} \omega_{n}\left(t_{1}, \ldots, t_{n}\right) & =\omega_{n+1}\left(t_{1}, \ldots, t_{i-1}, 1, t_{i}, \ldots, t_{n}\right) \\
\sigma^{i} \omega_{n}\left(t_{1}, \ldots, t_{n}\right) & =\omega_{n-1}\left(t_{1}, \ldots, t_{i-1}, \min \left\{t_{i}, t_{i+1}\right\}\right. \\
& \left.t_{i+2}, \ldots, t_{n}\right) \\
\sigma^{0} \omega_{n}\left(t_{1}, \ldots, t_{n}\right) & =\omega_{n-1}\left(t_{2}, \ldots, t_{n}\right) \\
\sigma^{n} \omega_{n}\left(t_{1}, \ldots, t_{n}\right) & =\omega_{n-1}\left(t_{1}, \ldots, t_{n-1}\right) \\
\sigma^{0} \omega_{0}= & 1
\end{aligned}
$$

where $I:=\Delta^{1}$.

Remarks 4.1. (1) One way to think of this construction is that, if we start with an element $\omega \in E^{1}$ such that $\sigma^{0} \omega=1$, then there are $2^{n}$ 
elements generated by $\omega$ in each $E^{n+1}$. To see this correspondence, take a vector in $\{0,1\}^{n}$, then substitute " $\omega *$ " for each 0 , and " $\partial$ " for each 1 , adding a final $\omega$. These elements will be at the vertices of an $n$-cube, and $\omega_{n}$ is then a homotopy between them.

(2) Lada's definition of a strong homotopy algebra differs slightly in that it omits all of the degeneracy conditions except $\sigma^{0} \omega_{0}=1$. Our choices are made so that we work with normalized, rather than unnormalized, cochain complexes associated to a cosimplicial complex. Since these are homotopy equivalent, both constructions will yield weakly equivalent deformation functors, even if we remove all degeneracy conditions.

(3) In [13, Proposition 3.11] it is shown that MC has a precise homotopytheoretical interpretation as the derived functor associated to the functor sending an SDC $E$ and $A \in \mathcal{C}_{\Lambda}$ to the set $\operatorname{MC}_{E}(A)$ from Definition 3.2. In the scenario of Section 3, it follows from the results of [13] that for $A \in s \mathcal{C}_{\Lambda}, \mathrm{MC}_{E}(A)$ is the set of objects of the Segal space of strong homotopy bialgebras over the object being deformed.

Proposition 4.1. $\mathrm{MC}_{E}: s \mathcal{C}_{\Lambda} \rightarrow$ Set is quasi-smooth. Moreover, if $f: E \rightarrow$ $F$ is a morphism of SDCs such that the maps $f^{n}: E^{n} \rightarrow F^{n}$ are smooth for all $n$, then $\mathrm{MC}_{E} \rightarrow \mathrm{MC}_{F}$ is quasi-smooth.

Proof. This follows immediately from [13, Lemma 3.9].

Definition 4.2. By Pridham [10, Lemma 1.5], $E^{0}$ is a group, which we denote by $G_{E}$. Observe that $G_{E}$ acts on $\mathrm{MC}_{E}$ by $(g, \omega) \mapsto g * \omega * g^{-1}$. We now define the deformation functor $\operatorname{Def}_{E}: s \mathcal{C}_{\Lambda} \rightarrow \mathbb{S}$ by $\operatorname{Def}_{E}:=\left[\underline{\mathrm{MC}}_{E} / \underline{G}_{E}\right]$, for $\underline{X}$ as in Definition 1.12, and $[-,-]$ the homotopy quotient of Section 1.4.

Proposition 4.2. If $A \in \mathcal{C}_{\Lambda}$, then $\operatorname{Def}_{E}(A)$ is just the classifying space $B \mathfrak{D} \mathfrak{e f} \mathfrak{f}_{E}(A) \in \mathbb{S}$ of the deformation groupoid $\mathfrak{D e f}_{E}(A)$ from Definition 3.2.

Proof. Take $\underline{\omega} \in \mathrm{MC}_{E}(A)$. Since $A \in \mathcal{C}_{\Lambda}, A^{K}=A$ for all connected simplicial sets $K$, so $E^{n+1}\left(A^{I^{n}}\right)=E^{n+1}(A)$, and $\omega_{n}=\omega_{0}^{*(n+1)}$, with the MaurerCartan relations reducing to

$$
\partial^{1} \omega_{0}=\omega_{0} * \omega_{0}, \quad \sigma^{0} \omega_{0}=1
$$

These are precisely the conditions defining the Maurer-Cartan space $\mathrm{MC}_{E}$ $(A)$ of Definition 3.2 , and $\mathfrak{D e f}_{E}(A)$ is the groupoid given by the action of $E^{0}(A)$ on $\mathrm{MC}_{E}(A)$, as required. 
Proposition 4.3. The functor $\operatorname{Def}_{E}$ is quasi-smooth. More generally, if $f: E \rightarrow F$ is a morphism of SDCs, such that $f^{n}: E^{n} \rightarrow F^{n}$ is smooth for all $n$, then $\operatorname{Def}_{E} \rightarrow \operatorname{Def}_{F}$ is quasi-smooth.

Proof. This follows immediately from Corollary 0.1 .

Proposition 4.4. The cohomology groups $H^{j}\left(\operatorname{Def}_{E}\right)$ are isomorphic to the groups $H^{j+1}(E)$ from Definition 3.3.

Proof. This follows immediately from [13, Corollary 4.13].

\subsection{Deformations of morphisms}

The problem that we now wish to consider is that of deforming a morphism with fixed endpoints. Assume that we have a category-valued functor $\mathcal{D}$ : $\mathcal{C}_{\Lambda} \rightarrow$ Cat. Fix objects $D, D^{\prime}$ in $\mathcal{D}(\Lambda)$, and a morphism $f$ in $\mathcal{D}(k)$ from $D$ to $D^{\prime}$. The deformation problem which we wish to consider is to describe, for each $A \in \mathcal{C}_{\Lambda}$, the set of morphisms $f_{A}: D \rightarrow D^{\prime}$ in $\mathcal{D}(A)$ deforming $f$. This amounts to taking the special case $\mathbb{I}=(0 \rightarrow 1)$ and $\mathbb{J}=\{0,1\}$ in Section 3.3.

Now assume that we have a diagram of functors from $\mathcal{C}_{\Lambda}$ to Cat as in Section 3, and consider the cosimplicial complex $F^{\bullet}$ in Sp given by $F^{\bullet}:=$ $E_{\mathcal{D} / \mathcal{B}}^{\bullet}(f)$ from Lemma 3.1 .

On $s \mathcal{C}_{\Lambda}$, we now define a deformation functor

$$
\operatorname{Def}_{F}(A) \subset \prod_{n \geq 0} \underline{F}^{n}(A)^{\Delta^{n}},
$$

associated to $F$, to consist of those $\underline{\theta}$ satisfying:

$$
\begin{aligned}
& \partial^{i} \theta_{n}=\epsilon_{n+1-i}^{*} \theta_{n+1}, \\
& \sigma^{i} \theta_{n}=\eta_{n-1-i}^{*} \theta_{n-1},
\end{aligned}
$$

for face maps $\epsilon_{i}: \Delta^{n} \rightarrow \Delta^{n+1}$ and degeneracy maps $\eta_{i}: \Delta^{n} \rightarrow \Delta^{n-1}$ defined as in [18, Chapter 8.]

Proposition 4.5. $\operatorname{Def}_{F}$ is quasi-smooth, and $H^{i}\left(\operatorname{Def}_{F}\right) \cong H^{i}(F)$.

Proof. The first statement follows from [3, Section VII.5], which shows that the total space functor Tot from cosimplicial simplicial sets to simplicial sets is right Quillen. The description of cohomology is straightforward. 
Proposition 4.6. If $\mathbb{I}$ is the category $(0 \stackrel{m}{\longrightarrow} 1)$, let $\mathbb{D}: \mathbb{I} \rightarrow \mathcal{D}(k)$ be the functor given by $\mathbb{D}(0)=D, \mathbb{D}(1)=D^{\prime}$ and $\mathbb{D}(m)=f$, then there is a canonical weak equivalence

$$
\operatorname{Def}_{F} \simeq \operatorname{Def}\left(E_{\mathcal{D} / \mathcal{B}}(\mathbb{D}) \times_{E_{\mathcal{D} / \mathcal{B}}(D) \times E_{\mathcal{D} / \mathcal{B}}\left(D^{\prime}\right)} \bullet\right)
$$

where $\bullet E_{\mathcal{D} / \mathcal{B}}(D) \times E_{\mathcal{D} / \mathcal{B}}\left(D^{\prime}\right)$ is defined by the object $\left(D, D^{\prime}\right) \in \operatorname{MC}\left(E^{\bullet}\right.$ $\left.(D) \times E^{\bullet}\left(D^{\prime}\right)\right)(\Lambda)$.

Thus $\operatorname{Def}_{F}$ governs deformations of $f$ which fix $D, D^{\prime}$.

Proof. Let $C:=E_{\mathcal{D} / \mathcal{B}}(\mathbb{D}) \times_{E_{\mathcal{D} / \mathcal{B}}(D) \times E_{\mathcal{D} / \mathcal{B}}\left(D^{\prime}\right)} \bullet$. By Pridham [13, Lemma 5.10], there are canonical equivalences $\underline{\operatorname{MC}}(C)(A) \simeq \operatorname{Def}(F)(A)$, so we need only observe that $C^{0}=1$, so $\operatorname{Def}(C)=\underline{\mathrm{MC}}(C)$. The final statement then follows from Proposition 3.3.

4.1.1. Deforming identity morphisms If we now consider deformations of the morphism $\operatorname{id}_{D}: D \rightarrow D$, write $F$ for the cosimplicial complex $E_{\mathcal{D} / \mathcal{B}}^{\bullet}\left(\mathrm{id}_{D}\right)$ governing deformations of $\mathrm{id}_{D}$, and $E$ for the SDC describing deformations of $D$, as defined in [11, Section 2] (or just by taking the special case $\mathbb{I}=\bullet$ of Definition 3.9). Note that $E^{n}=F^{n}$, with the operations agreeing whenever they are defined on both. If we write $e:=\partial^{0} 1 \in F^{1}$, note that we also have $\partial^{0} f=e * f$ and $\partial^{n+1} f=f * e$ for $f \in F^{n}$.

This gives us an isomorphism $C^{\bullet}(E) \cong C^{\bullet}(F)$, and hence $H^{n}$ (Def $\left._{E}\right)=$ $H^{n+1}(E) \cong H^{n+1}\left(\operatorname{Def}_{F}\right)$.

Proposition 4.7. Under the scenario above, the simplicial set $\operatorname{Def}_{F}(A)$ is weakly equivalent to the loop space $\Omega_{\operatorname{Def}_{E}}(A)$ of $\operatorname{Def}_{E}(A)$ over the point $\omega_{D} \in \operatorname{Def}_{D}(\Lambda)$. This equivalence is functorial in $A \in s \mathcal{C}_{\Lambda}$.

Proof. Define the SDC PE to be the fibre of $\mathrm{ev}_{0}: E^{I} \rightarrow E$ over the constants $\left\{e^{n}\right\}$. It follows from Lemma 3.2 that the cohomology groups of $P E$ are all 0 . Now define the SDC $\Omega E$ to be the fibre of ev $1: P E \rightarrow E$ over $\left\{e^{n}\right\}$.

By Proposition 4.6, $\operatorname{Def}_{F}$ is weakly equivalent to $\operatorname{Def}(\Omega E)$. By Proposition 4.3, $\operatorname{Def}_{P E} \rightarrow \operatorname{Def}_{E}$ is quasi-smooth, and the fibre is Def $\Omega E$. Since $\operatorname{Def}_{P E}$ is contractible, this means that $\operatorname{Def}_{\Omega E}$ is homotopic to the loop space of $\operatorname{Def}_{E}$.

Remark 4.2. Note that we can describe $\Omega E$ entirely in terms of the structure on $F$, since

$$
(\Omega E)^{n}=\left(F^{n}\right)^{n}
$$


with

$$
\begin{aligned}
\partial^{i}\left(f_{1}, \ldots, f_{n}\right) & =\left(\partial^{i} f_{1}, \partial^{i} f_{2}, \ldots, \partial^{i} f_{i}, \partial^{i} f_{i}, \ldots, \partial^{i} f_{n}\right), \\
\sigma^{i}\left(f_{1}, \ldots, f_{n}\right) & =\left(\sigma^{i} f_{1}, \sigma^{i} f_{2}, \ldots, \sigma^{i} f_{i}, \sigma^{i} f_{i+2}, \ldots, \sigma^{i} f_{n}\right), \\
\left(g_{1}, \ldots, g_{m}\right) *\left(f_{1}, \ldots f_{n}\right) & =\left(g_{1} * e^{n}, \ldots, g_{m} * e^{n}, e^{m} * f_{1}, \ldots e^{m} * f_{n}\right) .
\end{aligned}
$$

Now, given any smooth object $F \in c \mathrm{Sp}$, we may regard $F$ as a cosimplicial complex of smooth objects in Sp (as in [14, Definition 1.2.]), and then

$$
\underline{F}=\operatorname{Def}(F) \simeq \operatorname{Def}(\Omega E)
$$

This means that we cannot expect derived deformation functors coming from SDCs to have any more structure than arbitrary deformation functors.

Remark 4.3. In the case of Hochschild cohomology, the deformation functor of a morphism $R \stackrel{f}{\rightarrow} S$ of associative algebras can be defined over the category of Artinian associative algebras, rather than just $\mathcal{C}_{\Lambda}$. This means that the Lie bracket $H^{i}(f) \times H^{j}(f) \rightarrow H^{i+j+1}(f)$ defined in [14, Section 5.2 ] extends to an associative cup product. If $f=\mathrm{id}_{R}$ is an identity, then we know that the Lie bracket vanishes (since $\operatorname{Def}_{f}$ is a loop space, by Proposition 4.6), which is why the cup product becomes commutative. Of course, we also have the bracket $H^{i}\left(\mathrm{id}_{R}\right) \times H^{j}\left(\mathrm{id}_{R}\right) \rightarrow H^{i+j}\left(\mathrm{id}_{R}\right)$ associated to the deformation functor of the object $R$.

\section{Comparison with $[11]$}

Now assume that $\Lambda=k$, a field of characteristic 0. In [14], an equivalence was given between the homotopy category of $\mathbb{Z}$-graded DGLAs and $\mathrm{Ho}(s c \mathrm{Sp})$. Under the equivalences of Theorems 1.7 and 1.5, this equivalence sends a DGLA to its associated deformation functor in the sense of [9] (by Pridham [14, Remark 4.46]). By Pridham [14, Proposition 3.27], the corresponding functor from $d g \mathcal{C}_{k}$ to $\mathbb{S}$ is equivalent to Hinich's simplicial nerve:

Definition 5.1. Given a DGLA $L$, recall from [5, Definition 8.1.1] that the simplicial nerve $\Sigma(L): d g \mathcal{C}_{k} \rightarrow \mathbb{S}$ is defined by

$$
\Sigma(L)(A)_{n}:=\mathrm{MC}\left(L \otimes \mathscr{A}_{n}\right)(A)
$$

where $\mathscr{A}_{n}$ is is the algebra of polynomial differential forms on the standard $n$-simplex $\Delta^{n}$ (denoted $\Omega_{n}$ in $\left.[5]\right)$. 
However, in [11], a functor $\mathcal{E}$ was constructed from $\mathbb{N}_{0}$-graded DGLAs to SDCs, and Definition 4.2 then gives us an associated object of $s c \mathrm{Sp}$. The purpose of this section is to show that the two constructions are consistent with each other.

\subsection{DGLAs}

Definition 5.2. A DGLA is a graded vector space $L=\oplus_{i} L^{i}$ over $k$, equipped with operators [,] $: L \times L \rightarrow L$ bilinear and $d: L \rightarrow L$ linear, satisfying:

(1) $\left[L^{i}, L^{j}\right] \subset L^{i+j}$.

(2) $[a, b]+(-1)^{\bar{a} \bar{b}}[b, a]=0$.

$(3)(-1)^{\bar{c} \bar{a}}[a,[b, c]]+(-1)^{\bar{a} \bar{b}}[b,[c, a]]+(-1)^{\bar{b} \bar{c}}[c,[a, b]]=0$.

(4) $d\left(L^{i}\right) \subset L^{i+1}$.

(5) $d \circ d=0$.

(6) $d[a, b]=[d a, b]+(-1)^{\bar{a}}[a, d b]$.

Here $\bar{a}$ denotes the degree of $a, \bmod 2$, for $a$ homogeneous.

A DGLA is said to be nilpotent if the lower central series $\Gamma_{n} L$ (given by $\left.\Gamma_{1} L=L, \Gamma_{n+1} L=\left[L, \Gamma_{n} L\right]\right)$ vanishes for $n \gg 0$.

Definition 5.3. Given a nilpotent Lie algebra $\mathfrak{g}$, define $\hat{\mathcal{U}}(\mathfrak{g})$ to be the prounipotent completion of the universal enveloping algebra of $\mathfrak{g}$, regarded as a pro-object in the category of algebras. As in [15, Appendix A], this is a pro-Hopf algebra, and we define $\exp (\mathfrak{g}) \subset \hat{\mathcal{U}}(\mathfrak{g})$ to consist of elements $g$ with $\varepsilon(g)=1$ and $\Delta(g)=g \otimes g$, for $\varepsilon: \hat{\mathcal{U}}(\mathfrak{g}) \rightarrow k$ the augmentation (sending $\mathfrak{g}$ to $0)$, and $\Delta: \hat{\mathcal{U}}(\mathfrak{g}) \rightarrow \hat{\mathcal{U}}(\mathfrak{g}) \otimes \hat{\mathcal{U}}(\mathfrak{g})$ the comultiplication.

Since $k$ is assumed to have characteristic 0, exponentiation gives an isomorphism from $\mathfrak{g}$ to $\exp (\mathfrak{g})$, so we may regard $\exp (\mathfrak{g})$ as having the same elements as $\mathfrak{g}$, but with multiplication given by the Campbell-Baker-Hausdorff formula.

Definition 5.4. Given a nilpotent DGLA $L^{\bullet}$, define the Maurer-Cartan set by

$$
\operatorname{MC}(L):=\left\{\omega \in L^{1} \mid d \omega+\frac{1}{2}[\omega, \omega]=0 \in L^{2}\right\} .
$$


Define the gauge group $\operatorname{Gg}(L)$ by $\operatorname{Gg}(L):=\exp \left(L^{0}\right)$, which acts on $\mathrm{MC}(L)$ by the gauge action

$$
g(\omega)=g \cdot \omega \cdot g^{-1}-d g \cdot g^{-1}
$$

where denotes multiplication in the universal enveloping algebra of $L$. That $g(\omega) \in \mathrm{MC}(L)$ is a standard calculation (see [7] or [8]).

Definition 5.5. A morphism $f: L \rightarrow M$ of DGLAs is said to be a quasiisomorphism if $H^{*}(f): H^{*}(L) \rightarrow H^{*}(M)$ is an isomorphism.

Proposition 5.1. There is a model structure on the category of $\mathbb{Z}$-graded DGLAs, in which weak equivalences are quasi-isomorphisms, and fibrations are surjections. This category is Quillen-equivalent to the model category sDGSp of Definition 1.20.

Proof. See [14, Lemma 3.24 and Corollary 4.57].

\subsection{Cosimplicial groups}

Definition 5.6. Given an $\mathbb{N}_{0}$-graded DGLA $L$, let $D L$ be its denormalization. This becomes a cosimplicial Lie algebra via the Eilenberg-Zilber shuffle product. Explicitly

$$
D^{n} L:=\bigoplus_{\substack{m+s=n \\ 1 \leq j_{1}<\cdots<j_{s} \leq n}} \partial^{j_{s}} \ldots \partial^{j_{1}} L^{m}
$$

where we define the $\partial^{j}$ and $\sigma^{i}$ using the simplicial identities, subject to the conditions that $\sigma^{i} L=0$ and $\partial^{0} v=d v-\sum_{i=1}^{n+1}(-1)^{i} \partial^{i} v$ for all $v \in L^{n}$.

We now have to define the Lie bracket $\llbracket-,-\rrbracket$ from $D^{n} L \otimes D^{n} L$ to $D^{n} L$. Given a finite set $I$ of strictly positive integers, write $\partial^{I}=\partial^{i_{s}} \ldots \partial^{i_{1}}$, for $I=\left\{i_{1}, \ldots i_{s}\right\}$, with $1 \leq i_{1}<\cdots<i_{s}$. The Lie bracket is then defined on the basis by

$$
\llbracket \partial^{I} v, \partial^{J} w \rrbracket:= \begin{cases}\partial^{I \cap J}(-1)^{(J \backslash I, I \backslash J)}[v, w], & |v|=|J \backslash I|,|v|=|I \backslash J|, \\ 0 & \text { otherwise, }\end{cases}
$$

where for disjoint sets $S, T$ of integers, $(-1)^{(S, T)}$ is the sign of the shuffle permutation of $S \sqcup T$ which sends the first $|S|$ elements to $S$ (in order), and the remaining $|T|$ elements to $T$ (in order). Note that this description only works for $0 \notin I \cup J$. 
Definition 5.7. Now recall from [11, Section 4.2], that the functor $\mathcal{E}$ : $D G \mathrm{LA} \rightarrow \mathrm{SDC}$ from $\mathbb{N}_{0}$-graded DGLAs to SDCs is defined by

$$
\mathcal{E}(L)^{n}(A)=\exp \left(D^{n}(L) \otimes \mathfrak{m}_{A}\right)
$$

making $\mathcal{E}(L)$ into a cosimplicial complex of group-valued functors. To make it an SDC, we must define a $*$ product. We do this as the Alexander-Whitney cup product

$$
g * h=\left(\partial^{m+n} \ldots \partial^{m+2} \partial^{m+1} g\right) \cdot\left(\partial^{0}\right)^{m} h,
$$

for $g \in \mathcal{E}(L)^{m}, h \in \mathcal{E}(L)^{n}$.

Definition 5.8. Given a cosimplicial simplicial group $G$, define $\underline{\operatorname{MC}}(G) \in \mathbb{S}$ by $\underline{\operatorname{MC}}(G) \subset \prod_{n \geq 0}\left(G^{n+1}\right)^{\Delta^{n}}$, satisfying the conditions of [12, Lemma 3.3], i.e., the elements $\omega_{n} \in\left(G^{n+1}\right)^{\Delta^{n}}$ satisfy

$$
\begin{aligned}
\partial_{i} \omega_{n} & = \begin{cases}\partial^{i+1} \omega_{n-1}, & i>0 \\
\left(\partial^{1} \omega_{n-1}\right) \cdot\left(\partial^{0} \omega_{n-1}\right)^{-1}, & i=0\end{cases} \\
\sigma_{i} \omega_{n} & =\sigma^{i+1} \omega_{n+1}, \\
\sigma^{0} \omega_{n} & =1
\end{aligned}
$$

Define $\mathrm{MC}: s c \mathrm{Gp} \rightarrow$ Set by $\mathrm{MC}(G)=\underline{\mathrm{MC}}(G)_{0}$.

There is an adjoint action of $G^{0}$ on $\underline{\mathrm{MC}}(G)$, given by

$$
(g * \omega)_{n}=\left(\partial_{0}\left(\partial^{1}\right)^{n+1}\left(\sigma_{0}\right)^{n+1} g\right) \cdot \omega_{n} \cdot\left(\partial^{0}\left(\partial^{1}\right)^{n}\left(\sigma_{0}\right)^{n} g^{-1}\right),
$$

as in [12, Definition 3.8].

We then define $\operatorname{Del}(G)$ to be the homotopy quotient $\operatorname{Del}(G)=[\underline{\mathrm{MC}}(G) /$ $\left.G^{0}\right] \in \mathbb{S}$.

Let exp denote exponentiation of a nilpotent Lie algebra (giving a unipotent group).

Corollary 5.1. Given an $\mathbb{N}_{0}$-graded DGLA L, the deformation functor $\operatorname{Def}(\mathcal{E}(L)) \in s c \operatorname{Sp}$ is weakly equivalent to the functor

$$
A \mapsto \operatorname{Del}(\exp (D L \otimes \mathfrak{m}(A)))
$$

Proof. The $\mathrm{SDC} \mathcal{E}(L)$ corresponds to $A \mapsto \mathcal{E}(\exp (D L \otimes \mathfrak{m}(A))$ in the notation of $[13$, Section 3.1$]$, so the result is an immediate consequence of [13, Proposition 6.11]. 
Corollary 5.2. For $L$ as above, $\operatorname{Def}(\mathcal{E}(L))$ is weakly equivalent in the model category scSp to the functor $A \mapsto\left[\mathrm{MC}(\exp (D L \otimes \mathfrak{m}(A))) / \exp \left(L^{0} \otimes\right.\right.$ $\left.\left.\mathfrak{m}\left(A_{0}\right)\right)\right]$.

Proof. From [14, Lemma 2.26] $\mathrm{MC}(\exp (D L \otimes \mathfrak{m}(A))) \rightarrow \underline{\mathrm{MC}}(\exp (D L \otimes$ $\mathfrak{m}(A))$ ) defines a weak equivalence in scSp (although the former is not fibrant), and similarly for $L^{0} \otimes \mathfrak{m}\left(A_{0}\right) \rightarrow L^{0} \otimes \mathfrak{m}(A)$, so we get a weak equivalence on passing to the homotopy quotient.

\subsection{The final comparison}

Definition 5.9. Given an $\mathbb{N}_{0}$-graded DGLA $L$, define $\operatorname{Del}(L) \in s D G$ Sp to be the functor $\operatorname{Del}(L): d g \mathcal{C}_{k} \rightarrow \mathbb{S}$ given by the homotopy quotient

$$
A \mapsto\left[\mathrm{MC}\left(\operatorname{Tot}^{\Pi}(L \otimes N \mathfrak{m}(A))\right) / \exp \left(L^{0} \otimes \mathfrak{m}\left(A_{0}\right)\right]\right.
$$

with respect to the gauge action of Definition 5.4.

Corollary 5.3. Given an $\mathbb{N}_{0}$-graded DGLA L, the deformation functor $\operatorname{Def}(\mathcal{E}(L)) \in s c \operatorname{Sp}$ is weakly equivalent to $\mathbf{R S p f} N^{*} \operatorname{Del}(L)$, for $\mathbf{R S p f} N^{*}$ as in Theorem 1.6.

Proof. By Corollary 5.1, it suffices to show that the functors $\operatorname{Del}(L)$ and $A \mapsto \operatorname{Del}(\exp (D L \otimes \mathfrak{m}(A)))$ are weakly equivalent in $s c S p$. It follows from $[14$, Lemma 2.26] that the latter is weakly equivalent to

$$
A \mapsto\left[\mathrm{MC}(\exp (D L \otimes \mathfrak{m}(A))) / \exp \left(L^{0} \otimes \mathfrak{m}\left(A_{0}\right)\right)\right]
$$

which is not fibrant in general. Now, by [13, Theorem 6.23] this is isomorphic to

$$
A \mapsto\left[\mathrm{MC}\left(\operatorname{Tot}^{\Pi}(L \otimes N \mathfrak{m}(A))\right) / \exp \left(L^{0} \otimes \mathfrak{m}\left(A_{0}\right)\right]\right.
$$

which is just $\operatorname{Spf} N^{*} \operatorname{Del}(L)$. It follows from [14, Lemma 1.62] that $\mathbf{R S p f} N^{*}$ $F \cong \operatorname{Spf} N^{*} F$ for all levelwise quasi-smooth functors $F$.

Definition 5.10. Define $D G d g \mathcal{C}_{k}$ to be the category of Artinian local $\mathbb{N}_{0} \times \mathbb{N}_{0}$-graded graded-commutative $\Lambda$-algebras $A_{\bullet}^{\bullet}$ with differential of bidegree $(1,-1)$ and residue field $k$. Let $d g D G S p$ be the category of left-exact Set-valued functors on $D G d g \mathcal{C}_{k}$. 
Proposition 5.2. Under the equivalence of Proposition 5.1, an $\mathbb{N}_{0}$-graded $D G L A L$ corresponds to the deformation functor $\operatorname{Def}(\mathcal{E}(L)) \in s c \mathrm{Sp}$ of Definition 4.2.

Proof. The equivalence of [14, Corollary 4.57] is given by a functor $\mathbf{R S p f} D^{*}$ : $\mathrm{Ho}(s D G \mathrm{Sp}) \rightarrow \mathrm{Ho}(d g D G \mathrm{Sp})$, together with a functor

$$
\text { Spf } \operatorname{Tot}^{*} \mathrm{MC}: D G_{\mathbb{Z}} \mathrm{LA} \rightarrow d g D G \mathrm{Sp},
$$

on $\mathbb{Z}$-graded DGLAs, given by

$$
\operatorname{Spf} \operatorname{Tot}^{*} \operatorname{MC}(L)(A)=\operatorname{MC}(\operatorname{Tot} L \otimes \mathfrak{m}(A)) .
$$

By Corollary 5.3, it suffices to show that the objects $\mathbf{R S p f} D^{*} \operatorname{Del}(L)$ and Spf $\operatorname{Tot}^{*} \mathrm{MC}(L)$ are weakly equivalent in $d g D G \mathrm{Sp}$.

Taking $A \in D G d g \mathcal{C}_{k}$, it follows from the definitions that $\operatorname{Spf} D^{*} \operatorname{Del}(L)$ $(A)$ consists of maps $\operatorname{Spf}(D A) \rightarrow \mathrm{MC}(L) \times^{\exp \left(L^{0}\right)} W\left(\exp \left(L^{0}\right)\right)$ in $s D G \mathrm{Sp}$, where $D A \in\left(d g \mathcal{C}_{k}\right)^{\Delta}$ is defined by cosimplicial denormalization, and Spf $(D A) \in s D G \mathrm{Sp}$ is the functor $d g \mathcal{C}_{k} \rightarrow \mathbb{S}$ given in level $n$ by $\operatorname{Hom}_{d g \mathcal{C}_{k}}\left(D^{n} A,-\right)$.

Thus

$$
\operatorname{Spf} D^{*} \operatorname{Del}(L)(A) \subset \operatorname{MC}\left(\operatorname{Tot} L \otimes A^{0}\right) \times \operatorname{MC}\left(D\left(\exp \left(L^{0} \otimes A_{0}^{\bullet}\right)\right)\right.
$$

consists of pairs $(\omega, g)$ with $g * \partial_{D A}^{0} \omega=\partial_{D A}^{1} \omega$, corresponding in level $n$ to the map

$$
\begin{aligned}
\left(\operatorname{Spf} D^{n} A\right) & \rightarrow \operatorname{MC}(L) \times \exp \left(L^{0}\right)^{n}, \\
(\omega, g) & \mapsto\left(\omega,\left(\partial^{2}\right)^{n-1} g, \partial^{0}\left(\partial^{2}\right)^{n-2} g, \ldots,\left(\partial^{0}\right)^{n-1} g\right) .
\end{aligned}
$$

By Pridham [13, Theorem 6.23], we know that $\operatorname{MC}\left(D\left(\exp \left(L^{0} \otimes A_{0}^{\bullet}\right)\right) \cong\right.$ $\left.\mathrm{MC}\left(L^{0} \otimes A_{0}^{\bullet}\right)\right)$, giving us $\left.\gamma \in \mathrm{MC}\left(L^{0} \otimes A_{0}^{\bullet}\right)\right)$. Note that $g=\exp (\gamma)$, so

$$
g * \partial_{D A}^{0} \omega=\partial_{D A}^{0} \omega+[\gamma, \omega],
$$

with all higher terms vanishing, since $\sigma^{0} \gamma=0$, so $\llbracket \gamma, \llbracket \gamma, v \rrbracket \rrbracket=0$ for all $v$ (and in particular when $v=\partial_{D A}^{0} \omega$ ).

Thus we have

$$
\begin{gathered}
\operatorname{Spf} D^{*} \operatorname{Del}(L)(A)=\left\{(\omega, \gamma): \omega \in \operatorname{MC}\left(\operatorname{Tot} L \otimes A^{0}\right), \gamma \in \operatorname{MC}\left(L^{0} \otimes A_{0}^{\bullet}\right),\right. \\
\left.[\gamma, \omega]+d_{c, A} \omega=0\right\},
\end{gathered}
$$

where $d_{c, A}$ is the cochain differential on $A$. 
Now look at $\gamma+\omega \in(\operatorname{Tot}(L \otimes A))^{1}$. The equations combine to show that $\alpha:=\gamma+\omega$ lies in $\operatorname{MC}(\operatorname{Tot}(L \otimes A))$, since

$$
d \alpha+[\alpha, \alpha]=\left(d_{L} \omega+d_{A}^{s} \omega+[\omega, \omega]\right)+\left(d_{A} \gamma+[\gamma, \gamma]\right)+\left([\gamma, \omega]+d_{A, c} \omega\right)=0
$$

where $d_{A}^{s}$ is the chain differential on $A$.

Thus we have defined a map

$$
\psi: \operatorname{Spf} D^{*} \operatorname{Del}(L) \rightarrow \operatorname{Spf}\left(\operatorname{Tot}^{\Pi}\right)^{*} \operatorname{MC}(L)
$$

In fact, we have shown that

$$
\operatorname{Spf} D^{*} \operatorname{Del}(L)(A) \cong \operatorname{MCTot}\left(\left(L \otimes A^{0}\right) \times_{\left(L^{0} \otimes A^{0}\right)}\left(L^{0} \otimes A_{0}\right)\right)
$$

and [14, Lemma 4.13] then implies that $\psi$ is a weak equivalence (with similar reasoning to [14, Lemma 2.26]).

Finally, note that this gives cohomology groups (as defined in Definition 1.16) $H^{n}\left(\operatorname{Spf} D^{*} \operatorname{Del}(L)(A)\right) \cong H^{n+1}(L)$, and that

$$
H^{n}\left(\mathbf{R S p f} D^{*} \operatorname{Del}(L)\right) \cong H^{n}(\operatorname{Del}(L)) \cong H^{n+1}(L),
$$

since the equivalence of [14, Proposition 4.57] preserves cohomology groups. Therefore, the morphism $\operatorname{Spf} D^{*} \operatorname{Del}(L) \rightarrow \mathbf{R} \operatorname{Spf} D^{*} \operatorname{Del}(L)$ is also a weak equivalence by Corollary 1.2, and this completes the proof.

\subsection{An application}

Corollary 5.4. Let $X$ be a smooth scheme, for which there exists a flabby $D G L A$ resolution $\mathscr{L}^{\bullet}$ of the tangent sheaf $\mathscr{T}_{X}$. Then for $A \in s \mathcal{C}_{k}$, the simplicial nerve $\Sigma(L)(N A)$ of the $D G L A L^{\bullet}=\Gamma\left(X, \mathscr{L}^{\bullet}\right)$ is weakly equivalent to $\operatorname{Def}_{E}(A)$, where $E$ is the $S D C$ constructed in Example 3.1.1 (or Example 3.4 with $Y=\emptyset)$.

Proof. $\mathcal{E}\left(\mathscr{L}^{\bullet}\right)$ is a sheaf of SDCs on $X$, with $\Gamma\left(X, \mathcal{E}\left(\mathscr{L}^{\bullet}\right)\right)=\mathcal{E}(L)$. We may then form the Godement sheaf resolution $\mathscr{C}(\mathcal{E}(\mathscr{L}))$ of $\mathcal{E}(L)$, giving another sheaf of SDCs on $X$ (as in [11, Section 3.2]) and quasi-isomorphisms

$$
\mathcal{E}\left(\mathscr{L}^{\bullet}\right) \rightarrow \mathscr{C}\left(\mathcal{E}\left(\mathscr{L}^{\bullet}\right)\right) \leftarrow \mathscr{C}\left(\mathcal{E}\left(\mathscr{T}_{X}\right)\right)
$$


of flabby sheaves of SDCs on $X$, and hence quasi-isomorphisms

$$
\mathcal{E}(L) \rightarrow C(X, \mathcal{E}(L)) \leftarrow C\left(X, \mathcal{E}\left(\mathscr{T}_{X}\right)\right)
$$

of SDCs, where $C(X, \mathscr{F})=\Gamma(X, \mathscr{C}(\mathscr{F}))$ is the Godement resolution.

Finally, note that $\mathcal{E}\left(\mathscr{T}_{X}\right)(A)=\exp \left(\mathscr{T}_{X} \otimes \mathfrak{m}(A)\right)$ is the sheaf of infinitesimal algebra automorphisms of $\mathscr{O}_{X}$, so the methods of [11, Section 3.2.2] provide a quasi-isomorphism $C\left(X, \mathcal{E}\left(\mathscr{T}_{X}\right)\right) \rightarrow E$ of $\operatorname{SDCs}$. Thus $\operatorname{Def}(E) \simeq$ $\operatorname{Def}(\mathcal{E}(L))$, and Proposition 5.2 shows that this is equivalent to $L$ under the equivalences of Proposition 5.1.

\section{Acknowledgments}

This work was supported by Trinity College, Cambridge; and by the Engineering and Physical Sciences Research Council (grant number EP/ F043570/1).

\section{References}

[1] F.R. Cohen, T.J. Lada and J. Peter May, The homology of iterated loop spaces, Lect. Notes in Math., 533, Springer-Verlag, Berlin, 1976.

[2] D. Fiorenza and M. Manetti, L_o structures on mapping cones, Algebra Number Theory 1(3) (2007), 301-330, arXiv math.QA/0601312.

[3] P.G. Goerss and J.F. Jardine, Simplicial homotopy theory, Progress in Math., 174, Birkhäuser Verlag, Basel, 1999.

[4] A. Grothendieck, Technique de descente et théorèmes d'existence en géométrie algébrique. II Le théorème d'existence en théorie formelle des modules, in 'Séminaire Bourbaki', 5, Soc. Math. France, Paris, 1995, 369-390, Exp. No. 195.

[5] V. Hinich, DG coalgebras as formal stacks, J. Pure Appl. Algebra 162(2-3) (2001), 209-250

[6] L. Illusie, Complexe cotangent et déformations. I, Lect.e Notes in Math., 239, Springer-Verlag, Berlin, 1971

[7] M. Kontsevich, Topics in algebra - deformation theory, Lecture Notes, available at http://www.math.brown.edu/ abrmovic/kontsdef.ps, 1994. 
[8] M. Manetti, Deformation theory via differential graded Lie algebras, in Algebraic Geometry Seminars, 1998-1999 (Italian) (Pisa), Scuola Norm. Sup., Pisa, 1999, 21-48, arXiv math. AG/0507284.

[9] M. Manetti, Extended deformation functors, Int. Math. Res. Not. 2002(14) (2002), 719-756.

[10] J.P. Pridham, Deforming l-adic representations of the fundamental group of a smooth variety, J. Algebraic Geom., 15(3) (2006), 415-442

[11] J.P. Pridham, Deformations of schemes and other bialgebraic structures, Trans. Amer. Math. Soc. 360(3) (2008), 1601-1629

[12] J.P. Pridham, Pro-algebraic homotopy types, Proc. London Math. Soc. $97(2)$ (2008), 273-338, arXiv math. AT/0606107 v8.

[13] J.P. Pridham. The homotopy theory of strong homotopy algebras and bialgebras, Homol. Homotopy Appl., 12(2) (2010), 39-108, arXiv:0908.0116v2 [math.AG].

[14] J.P. Pridham, Unifying derived deformation theories, Adv. Math., 224(3) (2010), 772-826, arXiv:0705.0344v5 [math.AG].

[15] D. Quillen, Rational homotopy theory, Ann. of Math. 90(2) (1969), 205-295

[16] Z. Ran, Lie atoms and their deformations, Geom. Funct. Anal. 18(1) (2008), 184-221, arXiv:math/0412204v7.

[17] D.H. Van Osdol, Bicohomology theory, Trans. Amer. Math. Soc. 183 (1973), 449-476

[18] C.A. Weibel, An introduction to homological algebra, Cambridge University Press, Cambridge, 1994.

DPMmS, Centre for Mathematical Sciences

University of CAMBRIDGE

Wilberforce Road, Cambridge CB3 0WB

UK

E-mail address: J.P.Pridham@dpmms.cam.ac.uk

Received November 22, 2011 
